\title{
Perspective
}

PERSPECTIVE Actualité en histoire de l'art

$1 \mid 2021$

Portugal et espaces lusophones

\section{Itinérances artistiques et hybridations des formes dans les arts décoratifs au Portugal et au-delà des mers (XVI ${ }^{\mathrm{e}}-\mathrm{XVII}{ }^{\mathrm{e}}$ siècles)}

Artistic Odysseys and Hybrid Forms. The Decorative Arts in Portugal and beyond the Seas (16th - 17th Centuries)

Kunstwanderungen und die Herausbildung hybrider Formen im Kunstgewerbe in Portugal und Übersee (im 16. und 17. Jahrhundert)

Itinerâncias artísticas e hibridizações de formas nas artes decorativas em

Portugal e além-mar (séculos XVI-XVII)

Circolazioni artistiche e ibridazioni delle forme nelle arti decorative in Portogallo e oltremare (secoli XVI-XVII)

\section{Céline Ventura Teixeira}

\section{CpenEdition}

Journals

\section{Édition électronique}

URL : https://journals.openedition.org/perspective/23175

DOI : $10.4000 /$ perspective. 23175

ISSN : 2269-7721

Éditeur

Institut national d'histoire de l'art

\section{Édition imprimée}

Date de publication : 14 octobre 2021

Pagination : 132-154

ISBN : 978-2-917902-91-2

ISSN : $1777-7852$

Référence électronique

Céline Ventura Teixeira, «Itinérances artistiques et hybridations des formes dans les arts décoratifs au Portugal et au-delà des mers (xv1 $\left.\right|^{\mathrm{e}-\mathrm{xV} \|} \|^{\mathrm{e}}$ siècles) », Perspective [En ligne], 1 | 2021, mis en ligne le 31 décembre 2021, consulté le 13 mai 2022. URL : http://journals.openedition.org/perspective/23175 DOI : https://doi.org/10.4000/perspective. 23175 



\title{
Itinérances artistiques et hybridations des formes dans les arts décoratifs au Portugal et au-delà des mers (XVI $-\mathrm{XVII}$ siècles)
}

\author{
Céline Ventura Teixeira
}

Dès 1497, les navires de la Carreira da Índia ${ }^{1}$ ont assuré l'acheminement d'hommes et de marchandises de Lisbonne aux îles Ryūkyū. Ville à la croisée des mondes, Lisbonne se situe aussi à la croisée des savoir-faire tant les objets qui y sont échangés et les processus créatifs qui leur sont attachés sont divers. Entre les XVI ${ }^{e}$ et XVII ${ }^{e}$ siècles, les arts décoratifs connaissent un épanouissement singulier, représentatif des contacts établis dans les territoires ultramarins. Tandis que les ateliers indiens, chinois, japonais ou béninois reçoivent des commandes où la tradition locale se mêle aux formes européennes, les artisans portugais se confrontent à des ouvrages présentant de nouvelles techniques (porcelaines, laques, tapis) et à des matériaux exogènes (bézoards, coquillages, nacre ou écailles de tortue) qu'ils intègrent à leur tour dans leur production. De leur simple importation à leur réélaboration, ces " objets de mémoire ${ }^{2}$ " se situent à la rencontre de cultures visuelles et déploient de nouveaux langages aux formes recomposées. On peut alors déceler différents degrés de mobilité et de réception à travers ces phénomènes de circulation d'objets et de modèles décoratifs d'une sphère culturelle à une autre et interroger les processus de création et d'interprétation des modèles en jeu.

Cet essai s'attachera à mettre en lumière la nature plurielle et complexe des mécanismes de circulations artistiques entre les différents comptoirs établis par les Portugais et la couronne à l'aune d'études pionnières et des récentes recherches liées au contexte de la première globalité. Qu'ils soient issus d'une simple commande, offerts en cadeau diplomatique ou volés lors d'un pillage, ces objets d'art nourrissant les collections des monarques portugais sont aussi le reflet des relations politiques et des rapports de force qui ont noirci les pages de l'histoire portugaise. Loin d'adopter une vision uniforme et unilatérale, il s'agira d'appréhender les enjeux que supposent le mouvement de ces objets pèlerins et leur réception dans les différents univers d'accueil. La confection et l'envoi d'ouvrages et de matériaux révèlent des mécanismes de production qui sont à la fois déterminés par un goût et engagent la prise en considération des acteurs à l'origine de leur transport et de leur dissémination. Ces phénomènes circulatoires ne sont pas uniquement matériels mais aussi humains et formels, et participent de la diffusion de nouvelles techniques et de répertoires de modèles à l'origine d'une hybridation 
des formes tant ornementales que figuratives, perceptible dans les productions aussi bien portugaises qu'indiennes, chinoises, brésiliennes ou béninoises. Ces objets d'art et les expressions nées de ces rencontres constituent une mémoire vive qui matérialise des voyages entre les différentes parties du monde.

\section{État de la question}

À la suite des conquêtes de Goa et de Malacca en 1510 et 1511 , les navires chargés d'aromates, de gemmes indiennes, d'or et de soieries prennent le chemin de Lisbonne qui s'impose comme l'un des principaux nœuds de ce maillage commercial. Les ivoires africains et indiens, les porcelaines, les laques japonaises, les pierres précieuses et le cristal de roche de Ceylan achalandent les échoppes de la Rua Nova dos Mercadores, artère principale de la capitale (GSCHWEND, 2017). Lisbonne joue un rôle de premier ordre en tant que vitrine de biens, d'artefacts, d'objets en tout genre alimentant les collections et les répertoires décoratifs. Mise à l'honneur par le Museu nacional de arte antiga en 2017 dans une exposition intitulée " A cidade global: Lisboa no Renascimento ", la capitale lusitanienne synthétise les conséquences de ces circulations ultramarines à l'échelle locale. Marchands portugais mais aussi flamands, allemands, italiens et andalous dessinent les contours d'une ville cosmopolite où sont débarquées les merveilles d'Afrique, d'Orient, des Indes et d'Asie (GSCHWEND, LOWE, 2017a). Du local au global, différentes perspectives se déploient grâce à la mise en lumière d'un patrimoine important, éparpillé entre collections particulières et institutions muséales internationales.

Toutefois, les analyses développées autour du statut de cette " cité globale " engendrent l'usage et l'application de concepts sans véritable définition ou discussion. Lisbonne est encore désigné comme le " centre " d'un empire réunissant les continents africain, asiatique et américain. Or, qu'ils soient commerciaux, culturels ou symboliques, les réseaux établis outrepassent les frontières géographiques et politiques. Les notions de " centre " et de "périphérie " ne font plus sens dorénavant : Lisbonne fait partie d'un maillage commercial qui comprend déjà Séville, Anvers, Gênes et Venise. Elle jette un pont vers les nouvelles capitales commerciales émergeantes que sont Macao, Malacca ou encore Goa, dite aussi la "Rome d'Orient » ou " Goa dorée ». En retour, et par effet de miroir, Goa fut en l'occurrence confirmée dans son rôle de capitale de l'Estado da Índia d'où transitaient les marchandises (FLORES, 1998 ; SUBRAHMANYAM, [1993] 2013). Les échoppes de la ville donnaient à voir des ouvrages raffinés dont la confection était assurée par des orfèvres locaux, maîtrisant le travail de l'or et de l'argent. Ébénistes et tisserands étaient tout autant sollicités, notamment par les élites portugaises installées sur place (AHMED, 1991). À partir de 1580, Goa fait désormais partie d'un nouveau système commercial et politique ${ }^{3}$. L'union des couronnes portugaise et espagnole a entraîné la mise en place d'un réseau inédit de foyers artistiques qui comprenait Lisbonne, Porto, Séville, Medina del Campo, Madrid et Valladolid pour la péninsule Ibérique ; Pernambouc, Lima, Olinda, Mexico et Carthagène pour le Nouveau Monde ; Malacca, Macao, Nagasaki, Manille, Goa et Cochin pour l'Asie (SUBRAHMANYAM, [1993] 2013). Jusqu'alors dissociés, ces foyers sont dorénavant réunis. Composée de territoires aux systèmes politiques et culturels différents, cette " constellation ${ }^{4}$ " engendre plusieurs logiques et échelles combinatoires invitant à un changement de perspective. Par l'appréhension de ces mouvements, on passe d'une logique de grands ensembles à une logique de circulations individuelles qui met en relief un important réseau de migrations. Écrire cette histoire des arts décoratifs ne peut se fonder sur un seul point de vue mais sur une multiplicité de témoignages engageant leur confrontation et leur croisement. L'adoption 
d'une perspective xénologique impliquant un décentrement, une ouverture sur l'autre, permet de considérer ces objets d'art et leurs créateurs non plus selon une approche " exotique " mais égalitaire. Ces perspectives ne sont pas sans liens avec les recherches menées depuis une vingtaine d'années sur les enjeux de la première " globalité " abordant les phénomènes circulatoires, les acteurs ou les " passeurs culturels " (BÉNAT-TACHOT, GRUZINSKI, 2002) à l'origine de ces mouvements et les conséquences de ces mécanismes à l'échelle mondiale (SUBRAHMANYAM, [1993] 2013; $1994 ; 1997$; CHARTIER, 2001) . Dans son ouvrage Les quatre parties du monde, Serge Gruzinski souligne le rôle des prélats, des militaires, des missionnaires, des administrations et des artistes qui circulent et se confrontent à des sociétés plurielles où les rapports de forces ne sont pas les mêmes d'un continent à l'autre. Dans le cas des sociétés indiennes et japonaises, les Portugais sont maintenus en marge du pouvoir local. Les jeux de l'échange, du commerce et des processus de création sont dès lors définis suivant des termes imposés par la société d'accueil (GRUZINSKI, [2004] 2006).

L'étude des objets d'art en mouvement impliquait toutefois la définition d'un cadre théorique précis que Thomas DaCosta Kaufmann a proposé dans Toward a Geography of Art (DACOSTA KAUFMANN, 2004). Plus récemment, la publication de Circulations in the Global History of Art (DACOSTA KAUfMANN, Dossin, JoYeUX-PRUnel, 2015) a mis en évidence l'idée d'une nouvelle définition de l'histoire de l'art susceptible d'analyser ces phénomènes dans une perspective transculturelle. Les mouvements d'hommes, de matières et d'idées à l'époque moderne rendent compte d'une "vie des objets " qui concrétisent un dialogue entre les cultures. Déjà, Igor Kopytoff démontrait l'impact d'une époque sur la matérialité concrète ou symbolique des objets à même de produire des effets différenciés (KOPYTOFF, 1986). La relation entre la culture matérielle et la création de connexions à l'échelle mondiale se définit dans l'analyse d'objets tels que le textile, la porcelaine ou l'ivoire qui ont non seulement généré de nouvelles habitudes mais aussi de nouvelles pratiques de collection. L'ouvrage The Global Lives of Things: The Material Culture of Connections in the Early Modern World, dirigé par Anne Gerritsen et Giorgio Riello rassemble d'importantes contributions autour du rôle et de la signification plurielle des objets dans l'établissement des liens mondiaux entre 1400 et 1800 (GERRITSEN, RIELLO, 2015). Cette accumulation de significations que les objets acquièrent au fur et à mesure des déplacements a construit le cadre d'une réflexion poursuivie par nombre de chercheurs au cours de ces dernières années à l'image du numéro spécial du Journal of Early Modern History dirigé par Bronwen Wilson et Angela Vanhaelen « Making Worlds: Art, Materiality, and Early Modern Globalization » (VANHAELEN, WILSON, , 2019) où ce tournant matériel mondial ou global turn est étudié à l'aune des contextes politiques, des économies et des processus de création.

Si l'historiographie portugaise s'est penchée dès la fin du XIX ${ }^{e}$ siècle sur le patrimoine polymorphe et pluriel résultant des contacts établis avec ces territoires, il faut attendre les années 1970-1980 et les recherches de Maria Helena Mendes Pinto (MENDEs PINTO, 1990) pour qu'une nouvelle méthodologie se mette en place, fondée sur un décentrement géographique dans la lignée de ce que Pierre Chaunu qualifiait de "grand désenclavement planétaire " (CHAUNU, [1969] 1983) ${ }^{6}$. Les contributions de différents chercheurs étrangers ont également apporté un changement de perspective dans l'analyse de ces phénomènes. George Kubler s'intéressait déjà aux phénomènes de mélanges, de " métissages » des formes et d'hybridation des répertoires portugais, plaçant au premier plan une production artistique déconsidérée, car éloignée des grands foyers européens (KUBLER, 1959). L'impulsion donnée par Kubler, lorsqu'il définit ces problématiques sur les échanges artistiques et la construction de répertoires, engendra sur les scènes 
scientifiques portugaise et anglo-saxonne des analyses renouvelées sur la circulation de modèles européens par le biais de missionnaires, de négociants et d'artistes au Brésil et dans les différents pays d'Afrique et d'Asie. Initiée par Pedro Dias dans son ouvrage $A$ viagem das formas (DIAS, 1995), la question des transferts et des circulations artistiques a, peu à peu, été introduite dans les problématiques développées au Portugal à travers des cas précis (MENDONÇA, REBELO CORREIA, 2010). Au cours de ces dix dernières années, les expositions lisboètes illustrent la vitalité historiographique de ces questionnements : en collaboration avec le Smithsonian Institute, le Museu nacional de arte antiga a accueilli " Encompassing the Globe: Portugal e o mundo nos séculos XVI e XVII " en 2009, puis "A cidade global: Lisboa no Renascimento " en 2017 tandis que la Fundação Oriente a organisé « Jóias da Carreira da Índia » en 2014. À Paris, la récente exposition du musée Guimet, "Un firmament de porcelaines : de la Chine à l'Europe ", offrait l'opportunité de comprendre à travers la porcelaine les liens, tant diplomatiques que culturels, tissés entre la Chine et le Portugal (DÉLÉRY, TSAO, 2019). Les analyses se sont progressivement affinées en se concentrant sur des catégories d'objets comme la joaillerie ou l'orfèvrerie liturgique commandée par les missionnaires portugais aux ateliers indiens et chinois, donnant naissance à des ouvrages répondant à un modèle européen mais façonné par les artistes locaux (VASALlo E SILVA, 2000 ; BAILEY, 2013). Enfin, les chercheurs se sont focalisés sur une expression artistique particulière, qu'il s'agisse des arts du textile, de la question de la production indo-portugaise (HALlETT, PEREIRA, 2007 ; FERREIRA, 2014), des ivoires du Bénin (THORNTON, $1992 ; 2009$; MARK, 2010), des paravents japonais et du commerce de l'art Namban (MENDES PINTO, 1990 ; MARTINS, 2010 ; CURVELO, 2015) ou encore de la porcelaine et du mobilier de Chine (PINTO DE MATOS, 1994 ; NOBRE PAIS, 2012 ; BARRETO, SERRÃO, 2015).

\section{Des objets pèlerins, définitions et enjeux}

Dans son article intitulé "Lights on the Antipodes: Francisco de Holanda and an Art History of the Universal ", Alessandra Russo délivre une analyse sur les arts réalisés dans les " antipodes " - ces territoires jusqu'alors inconnus - à travers le témoignage de Francisco de Holanda ${ }^{7}$, figure incontournable de la Renaissance portugaise. Le regard qu'il porte sur les œuvres d'art produites en Afrique, en Asie et dans les Amériques redéfinit l'art sous une perspective universelle, abolissant l'idée de transmission d'excellence artistique (RUSSO, 2020). Les ivoires de Ceylan, les colchas da Índia, les éventails, la joaillerie, les porcelaines de Chine témoignent d'un savoir-faire inédit éveillant l'intérêt et se disséminant dans les sociétés européennes du XVI siècle.

Les ordonnances, les correspondances et les inventaires royaux ont permis d'identifier la commande ou l'envoi d'objets en guise de présent. D'une cour à l'autre, les relations politiques entre monarques ont également été déterminantes pour la diffusion d'ouvrages en provenance des différents comptoirs (DIAS, 1995). Les ambassades ont joué un rôle clé non seulement dans la consolidation des relations politiques mais aussi dans la diffusion et la naissance d'un goût. En 1542, le roi de Kōtte (actuel Sri Lanka), Bhuvanekabāhu VII (1521-1551), envoie par le biais de son chambellan, Sri Ramaraksa Pandita, un coffret en ivoire au roi dom João III (1502-1557). L'étude minutieuse établie par Zlotán Biedermann sur ce coffret révèle plusieurs facettes liées au rôle de cet objet (BIEDERMANN, 2017). Ce cadeau diplomatique venait également confirmer la vassalité ${ }^{8}$ du roi ceylanais envers la couronne portugaise, immortalisée dans les décors appliqués. Le prince Dharmapāla, petit-fils et successeur de Bhuvanekabāhu VII, y apparaît couronné par le monarque 
portugais, ce qui confirme non seulement la fidélité du souverain ceylanais au trône portugais mais aussi la protection accordée par dom João III. Ce coffret en ivoire n'est toutefois pas le seul présent envoyé à cette occasion. Les cuillères en cristal de roche serties de rubis, au même titre que les boucles $d^{\prime}$ oreilles et les bagues en provenance de Ceylan étaient très appréciées par l'épouse du roi, dona Catarina de Áustria (1507-1578)9. L'attrait de la reine pour la nacre, les ivoires ou les laques a déjà été largement mis en lumière (GSCHWEND, 1996). La salière en cristal de roche adoptant la forme d'un éléphant reflète ce goût naissant (fig. 1) au point qu'en 1545, Diogo Vaz, orfèvre personnel de la reine Catherine, s'embarque pour l'Inde avec João de Castro, le vice-roi nouvellement nommé, pour travailler depuis Ceylan sur des commandes destinées à la cour (GSCHWEND, LOWE, 2017b). Toutefois, ces cadeaux diplomatiques sont aussi le reflet de tensions et de rapports de force. À la mort de Bhuvanekabāhu VII, probablement orchestrée par le vice-roi des Indes Afonso de Noronha, le prince Dharmapāla se voit

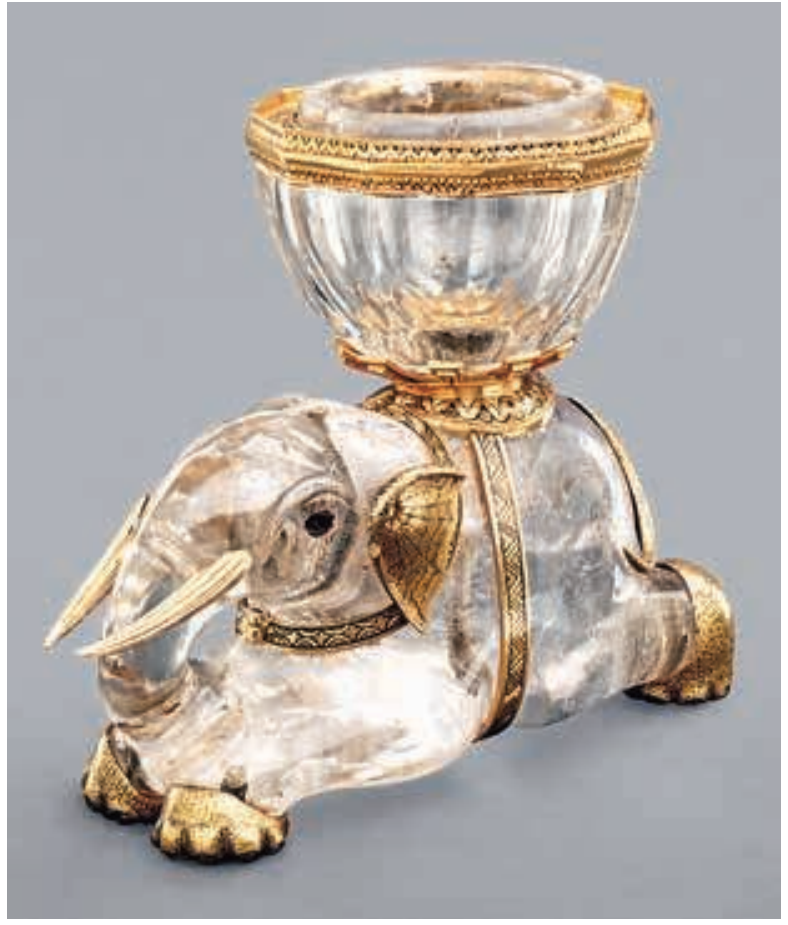

1. Atelier indien (Décan), Francisco Lopez (orfèvre, Lisbonne), Éléphant, vers 1550, cristal de roche, or et émail, 7,3 ×9,4 ×4,9 cm, Vienne, Kunsthistorisches Museum Wien, Kunstkammer, inv. n 2320. contraint de remettre le trésor royal contenant de rares pierres précieuses, de l'or, des bijoux et de la vaisselle suivant l'inventaire établi par le surintendant des finances de l'Estado da Índia, Simão Botelho (CRESPO, 2019). Ce même inventaire, publié une première fois par Sousa Viterbo en 1904, indique non seulement le contenu de ce trésor mais aussi les fruits de sa revente à Goa. Ce pillage a ainsi financé la sécurité des territoires et a été une manne importante de luxueux cadeaux diplomatiques, révélant les rapports de force liés à la colonisation des territoires et déterminant en partie les transactions et les circulations matérielles.

La dissémination de ces objets est également liée à l'attrait qu'ils ont exercé. Le plaisir de posséder des biens originaux pour orner les intérieurs ou les corps entre en corrélation avec un autre phénomène, lié à la réception de ces objets d'art. L'éventail japonais est en cela emblématique d'un changement d'usage. Initialement utilisé par les samouraïs, les shoguns et les daïmios, l'éventail devient un accessoire de distinction incontournable pour les dames de la cour, avec lequel elles parachèvent leur toilette. Son introduction est notamment due à la présence de Bernardo, disciple du jésuite François Xavier, et premier Japonais à se rendre sur le continent européen en 1552. Achetés au nombre de quarante par dona Catarina en 1561, les lequios, en provenance du Japon ou des îles Ryūkyū, étaient offerts aux favorites de la reine (GSCHWEND, 2017 ; ALFERES PINTO, 2018). Les portraits d'infantes et de princesses laissent entrevoir ce goût pour un tel atour. Doña Juana de Austria (1535-1573), María Manuela de Portugal (1527-1545) ou encore Isabel Clara Eugenia (1566-1633) participent notamment de cette diffusion entre les cours portugaise et espagnole, alors réunies sous un même sceptre. Aristocrates, nobles ou hauts dignitaires ne sont pas en reste. L'anecdote veut que le gouverneur de Goa, Afonso de Albuquerque (1453-1515), souhaitait envoyer 


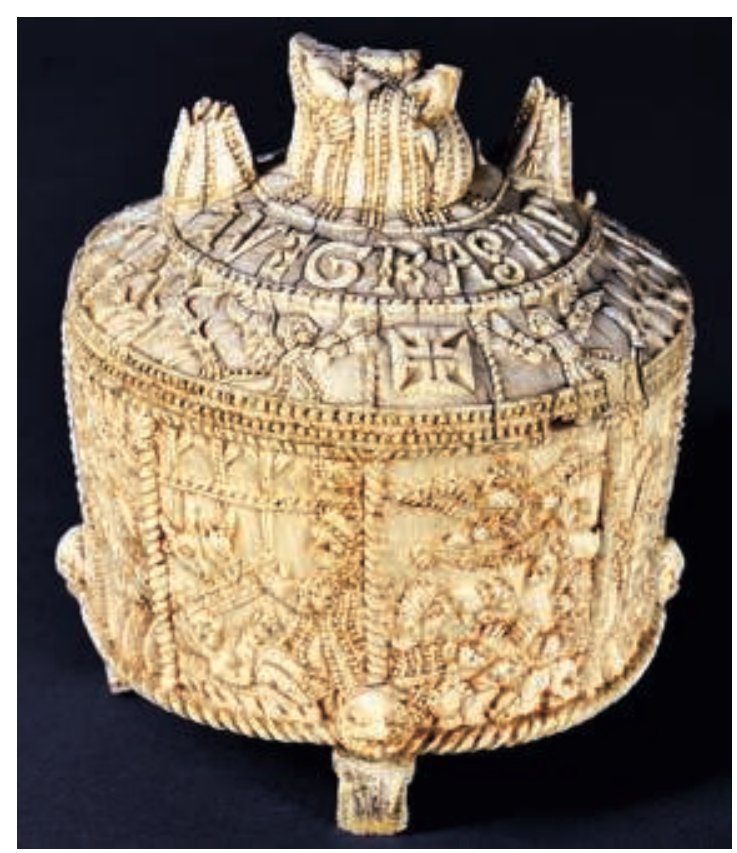

2. Atelier sapi, pyxide, 1500-1530, ivoire, $15,5 \times 8,5 \mathrm{~cm}$, Viseu, Museu nacional Grão Vasco, inv. n ${ }^{\circ} 1306$.

3. Atelier de Jingdezhen, Gomil (jarre), 1519-1521, dynastie Ming, porcelaine, 26,4 × 7,6 × 9,5 cm, Lisbonne, Casa-Museu Medeiros e Almeida, inv. FMA 814. des brodeuses à la cour de dona Maria afin que les panos da Índia, c'est-à-dire les couvertures, les jetés de lits et autres objets brodés soient produits à Lisbonne même (Albuquerque, [1577] 1973). Malheureusement, le navire La Flor de la Mar sombra, emportant avec lui les objets précieux et la main-d'œuvre. Mis au jour par Hugo Miguel Crespo, l'inventaire après décès de Simão de Melo Magalhães, capitaine de Malacca en 1545, révèle également la valeur tant matérielle que symbolique que suggèrent ces ouvrages (CRESPO, $2014 ; 2015)$. Si les porcelaines de Chine tiennent une place importante dans cet inventaire, les textiles asiatiques sont aussi largement représentés. On y trouve des soieries chinoises, des couvertures et des broderies d'Inde ; l'éventail de biens tissés englobe également vingt-quatre tapis indo-perses ${ }^{10}$. Le marché du textile constitue en effet une frange importante du commerce portugais ${ }^{11}$ dont les récentes études par Maria João Pacheco Ferreira ont mis en lumière les mécanismes d'usage et de réception, en particulier des colchas ou panos da Índia dans la société portugaise des $\mathrm{XVI}^{\mathrm{e}}$ et XVII ${ }^{\mathrm{e}}$ siècles ${ }^{12}$. Initialement considérés comme des objets de luxe, les tapis, les tissus brodés et les soieries ont progressivement été introduits dans l'espace religieux et civil au point qu'une pragmatique fut établie en 1609 afin de limiter l'acquisition et l'usage des panos da Índia par les différentes strates de la société portugaise (FERREIRA, 2016). La supériorité technique que présentent ces ouvrages a été une source de stimulation pour les ateliers portugais. Si, au niveau européen, les importations ne représentaient qu'un faible pourcentage, l'impact des textiles en provenance des Indes et de Chine ont eu néanmoins un véritable effet sur les goûts du moment (BERG, 2009).

Premier comptoir européen du golfe de Guinée, Mina ou Elmina tisse un lien avec Lisbonne dès les années 1490 à travers l'envoi d'objets raffinés. Les pyxides ou les salières de Sierra Leone suscitent l'intérêt du monarque et de la cour portugaise (BASSANI, 2000 ; MASSING, 2009 ; AFONSO, SILVA HORTA, 2013). Probablement réalisées par le peuple ashanti (appartenant aux Akans), ces pièces montrent non seulement le savoir-faire des artisans de cette région mais aussi les relations commerciales solides établies entre Lisbonne et Mina. Les programmes décoratifs déployés sur ces objets, où se mélangent technique locale et formes exogènes (TRNEK, VASSALO E SILVA, 2001 ; MASSING, 2009; MARK, 2009), sont des indicateurs de la présence portugaise. Les pyxides arborant de tels détails suggèrent l'adaptation d'un modèle imposé dans le cadre d'une commande. Reposant sur quatre lions, la pyxide du Museu nacional Grão Vasco (Viseu) unit le travail traditionnel de l'ivoire au modèle imposé (fig. 2). Le couvercle présente une inscription latine "Ave Grasias $P$ " accompagnée des armes de l'ordre du Christ et de la couronne portugaise. La vie de la Vierge est retracée dans les différentes sections composant le corps cylindrique de cette pyxide. Les encadrements perlés et enroulés ainsi que le travail détaillé de l'ivoire sont autant d'éléments résultant 


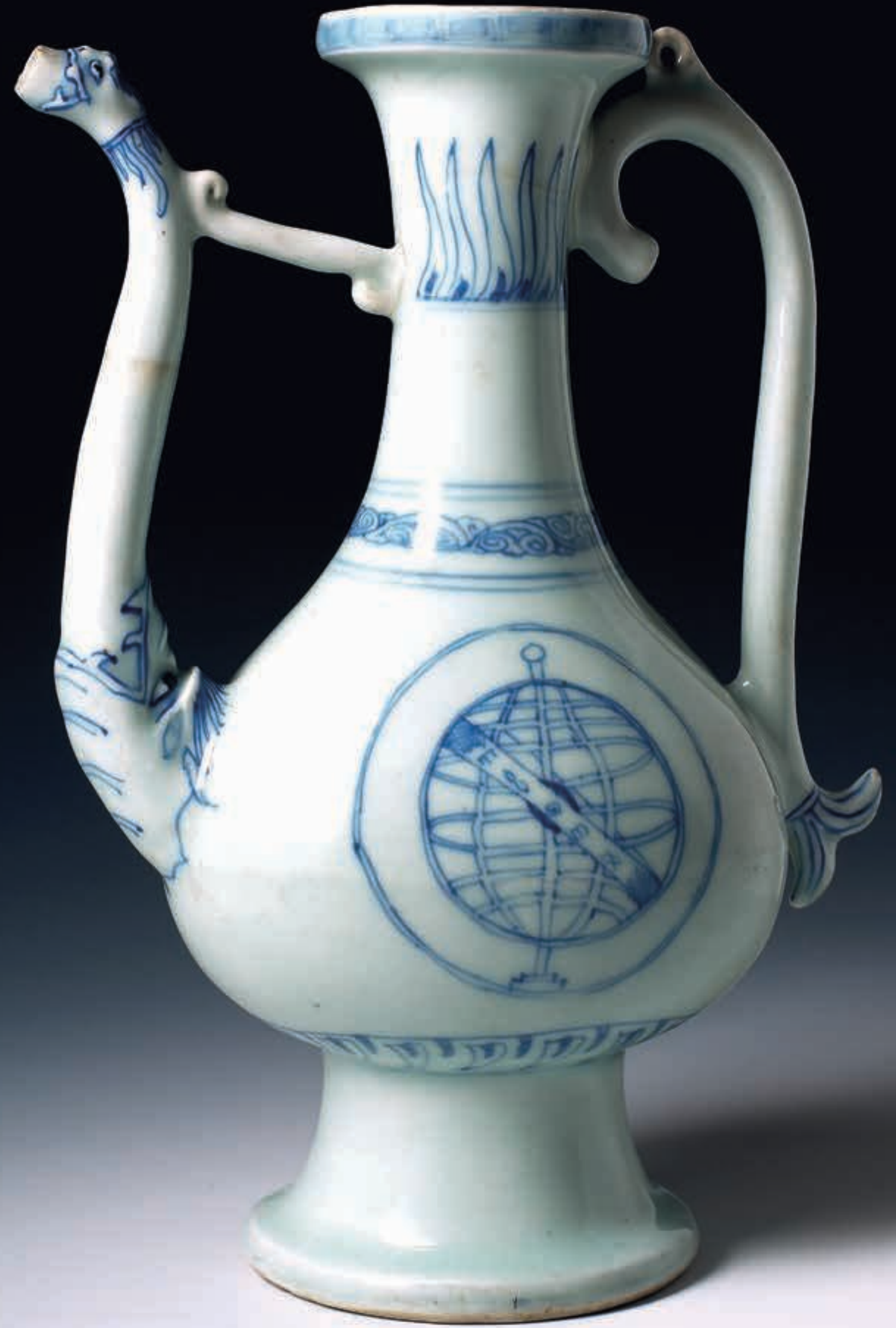




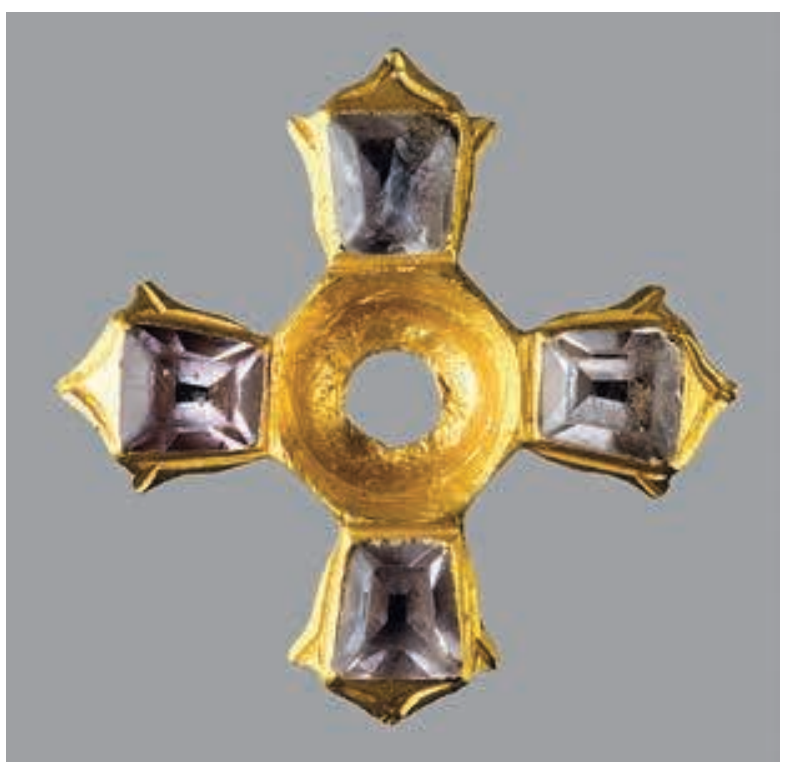

4. Atelier de Ceylan ou du Portugal, pendentif cruciforme, fin du XVI siècle, or et saphir, Lisbonne, Museu nacional de arqueologia, inv. $n^{\circ}$ CNANS 04673.01.004. de l'association de traditions visuelles inconnues de l'artisan et directement inspirées d'ouvrages gravés. Les objets commandés se devaient d'entrer en adéquation avec les attentes et de respecter les dogmes établis du milieu de leur réception. Introduits par les Portugais, les gravures, les livres d'heures ou encore les ouvrages tels que le Traité des saintes images de Molanus ou l'Evangelicae historiae imagines d'Ignace de Loyola ont été progressivement imposés comme source d'inspiration première des artisans sapis (MASSING, 2009).

Parmi les objets composant la collection royale au XVI ${ }^{\mathrm{e}}$ siècle, dom Manuel $\mathrm{I}^{\mathrm{er}}$ (14691521) manifesta une attention particulière pour les porcelaines de Chine, sur lesquelles les premiers travaux significatifs ont été initiés par Daisy Lion-Goldschmidt, Maria Antónia Pinto de Matos et Alexandre Nobre Pais. Arborant les armes de la couronne et la fameuse sphère armillaire, des quantités considérables de porcelaine de Jingdezhen furent acheminées jusqu'à Lisbonne depuis

Malacca (LION-GOLDSCHMIDT, 1984 ; PINTO DE MATOS, 1994 ; KRAHL, 2009 ; GERRITSEN, MCDOWALL, 2012). Dans un exemplaire conservé à la Casa-Museu Medeiros e Almeida (Lisbonne, fig. 3), le pinceau de l'artisan chinois unit tradition ornementale locale et motifs étrangers sur une même surface. Les pièces provenant du galion San Diego ${ }^{13}$, coulé en mer des Philippines en 1600, témoignent également du commerce intense des porcelaines de la période Wanli et des commandes passées par la couronne espagnole aux ateliers chinois, à l'instar des " bouteilles de Philippe II" (KRAHE, 2012-2013). Aux armes de la Castille et reposant sur un pied trapézoïdal, le corps circulaire aplati est surmonté d'un goulot cylindrique large et délicatement évasé (DÍAZ, 2010 ; CONDE Y CERVANTÉS, 2014). Le blason est ainsi entouré de fleurs de lotus et d'une végétation propre aux combinaisons déployées sur les porcelaines. L'élégance du dessin associée à la gamme de nuances de bleu de cobalt participe au raffinement de cette bouteille dont il ne demeure, à l'heure actuelle, qu'une dizaine d'exemplaires (DÍAZ, 2010 ; CONDE y CERVANTÉs, 2014). Cette catégorie d'objets produits par les ateliers chinois à destination du marché européen est l'une des multiples manifestations matérielles et artistiques où les formes occidentales côtoient les techniques et les répertoires de modèles extrême-orientaux.

La joaillerie d'Asie produit également des objets dont les métamorphoses sont directement liées au goût du moment. Réalisés dans le Sud de l'Inde ou à Ceylan, les pendeloques, bagues et autres bracelets datant du XVI e siècle sont aujourd'hui très rares. Soumises aux aléas du goût et des modes, ces pièces furent très souvent modifiées au fil du temps ou fondues si le contexte financier le nécessitait (CRESPO, 2017). Le commerce des pierres précieuses et des perles entre l'Asie et le Portugal a été très dynamique, en témoignent les quantités se déversant dans le port de Lisbonne qui ont approvisionné les ateliers d'orfèvre et de joailliers de la capitale. En provenance des mines de Golkonda (CRESPO, 2014), les gemmes et les diamants indiens ont été travaillés et remontés suivant 
d'autres techniques au Portugal. Se différenciant du savoir-faire kundan (GSCHWEND, 2015-2016) propre aux ateliers indiens et reconnaissables par un jeu d'incrustation des pierres, les travaux des ateliers portugais suivent quant à eux un modèle ornemental européen caractéristique de la période (fig. 4). Si les portraits de monarques et de nobles illustrent le goût et la recherche de ces gemmes, leur représentation permet aussi de formuler visuellement le façonnement matériel et artistique de ces pierres précieuses. Ce voyage au-delà des mers est également à l'origine de l'arrivée de matériaux méconnus appartenant aux règnes animal, organique, végétal et minéral. Bézoards, nacre, écaille de tortues, plumages, coquille de nautile, corne de rhinocéros sont autant de naturalia que les artisans européens « transforment " (FENNETAUX, MILLER BLAISE, ODDO, 2020). Leur essence première laisse place à un façonnement élaboré, propre aux artificialia. Ramenés pour leurs vertus médicinales, les cornes de rhinocéros étaient notamment utilisées en tant que remèdes contre la mélancolie, la peste ou l'épilepsie (GSWCHEND, 2017). La rareté de ces matériaux était également mise en valeur à travers leur réélaboration (fig. 5). Aux objets naturels présents dans les Kunstkammern s'ajoutaient des ouvres d'art révélant toute l'habileté et l'ingéniosité des orfèvres portugais installés parfois sur place ou dans la capitale ${ }^{14}$. L'étude comparée des productions portugaises incorporant les modèles venus d'Orient et des pièces réalisées dans les territoires de l'Empire enrichit le traitement des questions liées à la dimension transculturelle de ces objets d'art, tant dans leur matérialité que dans leur forme (KOPYTOFF, 1986). Ces « hybrides » sont le résultat d'un processus combinatoire où la rencontre de divers éléments donne naissance à un nouvel objet (BURKE, 2016).

Au sein de ce maillage, ces objets ne prenaient pas uniquement la route de Lisbonne mais transitaient également entre les territoires dépendants des couronnes portugaises et espagnoles. Les laques dites Namban sont particulièrement représentatives de ce phénomène (OlIVEIRA E COSTA, 1993 ; Aguiló Alonso, 1999 ; CURVELO, 2008 ; MARTINS, 2010). Les travaux d'Alexandra Curvelo autour des laques soulignent en ce sens la diffusion de l'art Namban dans les vice-royautés d'Amérique (CURVELO, 2008). D'abord utilisée par les Japonais pour identifier l'arrivée des premiers Européens, l'expression namban-ji (littéralement « barbares du sud ") a ensuite été employée pour désigner la rencontre culturelle et artistique de ces populations durant une période s'étendant de 1543 à 1639. La brillance intense de la laque japonaise provoqua un engouement tel que cette spécificité technique fut mise à profit (fig. 6). Les laques Namban furent alors produites pour l'exportation, et distinguées des produits destinés au commerce intérieur. On retrouve des traces de cette pratique artistique dans les vice-royautés d'Amérique

5. Atelier indo-portugais de Goa ou Cochin, coupe de rhinocéros, 1558-1565, corne de rhinocéros et vermeil, $21 \mathrm{~cm}$, Naples, Museo e Gallerie di Capodimonte, inv. $\mathrm{n}^{\circ} 10350-1870$ AM.

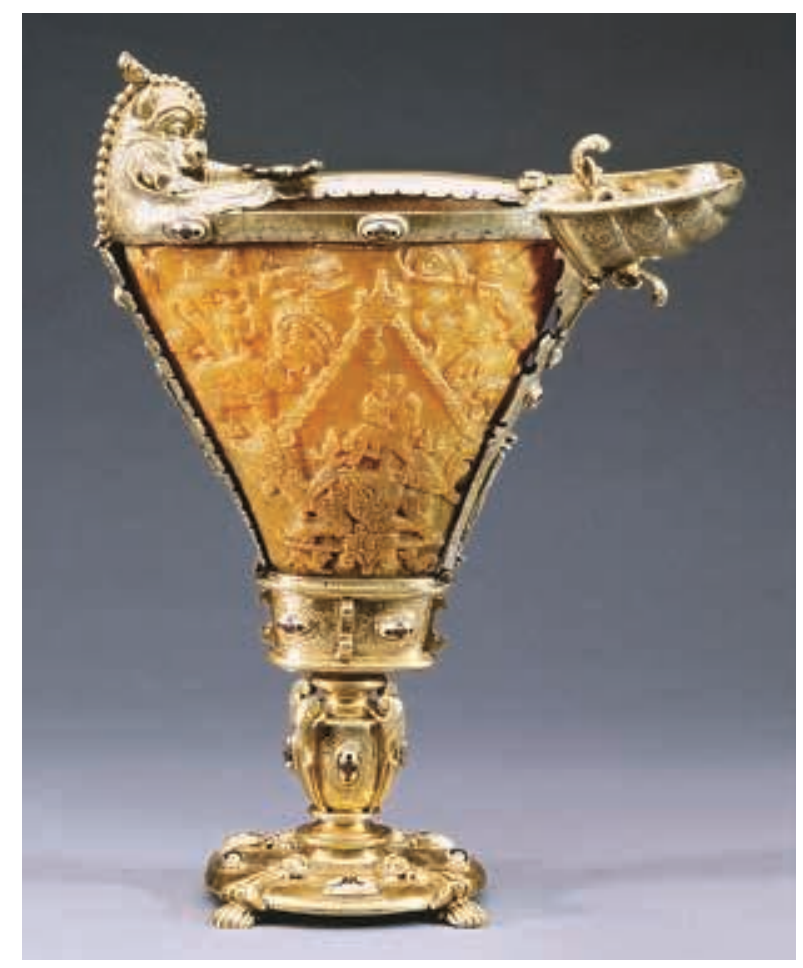


(LUTERBACHER, 2019), exprimant toute la valeur et l'originalité de ce phénomène de diffusion des formes. En sus des formes ibériques, viennent très largement s'ajouter les modèles asiatiques dans la production mexicaine. Les laques mexicaines aux origines précolombiennes sont aussi connues sous l'appellation de maque, expression qui fait écho au terme japonais maki-e-shi ( " l'artiste laqueur »), induisant par-là même une connaissance antérieure de la technique.

\section{Imitation, mélange et hybridité}

Les terminologies jusque-là employées pour qualifier les phénomènes d'interprétation des modèles, de reconstructions visuelles se sont révélées très souvent impropres. "Influence ", " assimilation ", " exotique " sont autant d'expressions qui laissent entendre un nivellement, une subalternité entre les acteurs à l'origine de ces productions.

L'interaction culturelle s'exprime dans l'échange de savoirs et l'adoption de techniques artistiques étrangères. Dès la fin du XVI ${ }^{\mathrm{e}}$ siècle, l'élégance des porcelaines de la période Wanli séduit et génère une forte demande à l'échelle européenne, devenant un véritable parangon ornemental et esthétique. L'impact des porcelaines sur la fabrication de faïence au Portugal trouve dans la thèse d'Alexandre Nobre Pais une analyse fine révélant plusieurs mécanismes d'imitation des répertoires figuratifs et ornementaux développés dans les porcelaines et adaptés sous le pinceau des faïenciers portugais (NOBRE PAIS, 2012). Si, de son côté, Cinta Krahe a étudié ce même phénomène dans la production espagnole (KRAHE,

2012-2013), l'analyse croisée des ateliers ibériques au temps de l'union dynastique n'est que peu étudiée. Depuis Lisbonne,

6. Atelier japonais, boîte, premier quart du XVII siècle, laque et feuilles d'or, $27 \times 24 \times 22 \mathrm{~cm}$, Lisbonne, Museu nacional de arte antiga, inv. $\mathrm{n}^{\circ} 68 \mathrm{Cx}$.

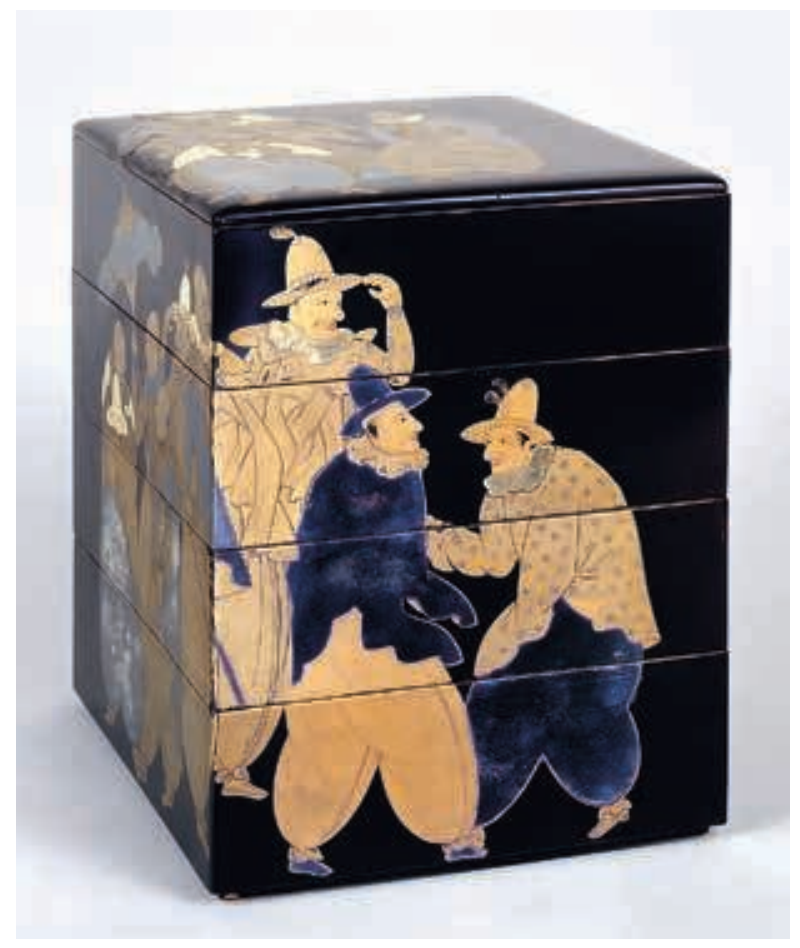
l'imitation de porcelaines chinoises se propage au point que certains faïenciers, tant portugais qu'espagnols, se spécialisent dans la vaisselle « à la mode de Chine " (VENTURA TEIXEIRA, 2019). La capitale portugaise affiche une production dynamique, comme en témoigne la soixantaine de fours existants au milieu du XVI siècle $^{15}$. À partir de 1592, le Livro de regimento dos officiais mecânicos, document régissant l'activité céramique, inclut un chapitre dédié aux règles et aux exigences requises dans le cadre de l'activité céramique (CORREIA, 1918a ; 1918b ; SANTOS SIMÕES, 1971 ; MANGUCCI, 1996 ; NOBRE PAIS, 2012 ; VENTURA TEIXEIRA, 2019). Il est ainsi précisé que tout artisan prétendant à la qualification de potier doit démontrer sa connaissance et son savoir-faire dans la réalisation d'objets de diverses catégories dont la porcelaine, bien que son secret ne soit alors toujours pas percé ${ }^{16}$. Ce phénomène s'explique en raison de la forte concurrence hollandaise dans le commerce des porcelaines dès le début du XVII ${ }^{\text {e }}$ siècle, auquel s'ajoutent les troubles dynastiques survenus en Chine 
autour des années 1640, et qui ont fortement perturbé les routes commerciales. Les manufactures de Jingdezhen ne parvenaient plus à acheminer leurs productions vers Malacca (CRICK, 2017). Parallèlement à ce contexte délicat, les faïenciers lisboètes et sévillans durent répondre à une demande grandissante. Leur clientèle ne se limitait plus aux cours princières mais comprenait également l'aristocratie et la bourgeoisie, lesquelles souhaitaient aussi posséder des similis de porcelaine (fig. 7). Les faïences confectionnées au Portugal et en Espagne présentent des décors évoquant les répertoires chinois. Pureté de la glaçure, qualité des émaux et diversité des motifs sont autant d'aspects qui caractérisent les faïences produites par les ateliers ibériques pour le marché intérieur mais également pour le marché extérieur ${ }^{17}$ (BAART, 1987). Cette production lisboète a été évoquée par le chroniqueur João Baptista Lavanha dans le récit qu'il fit de l'entrée royale de Philippe III

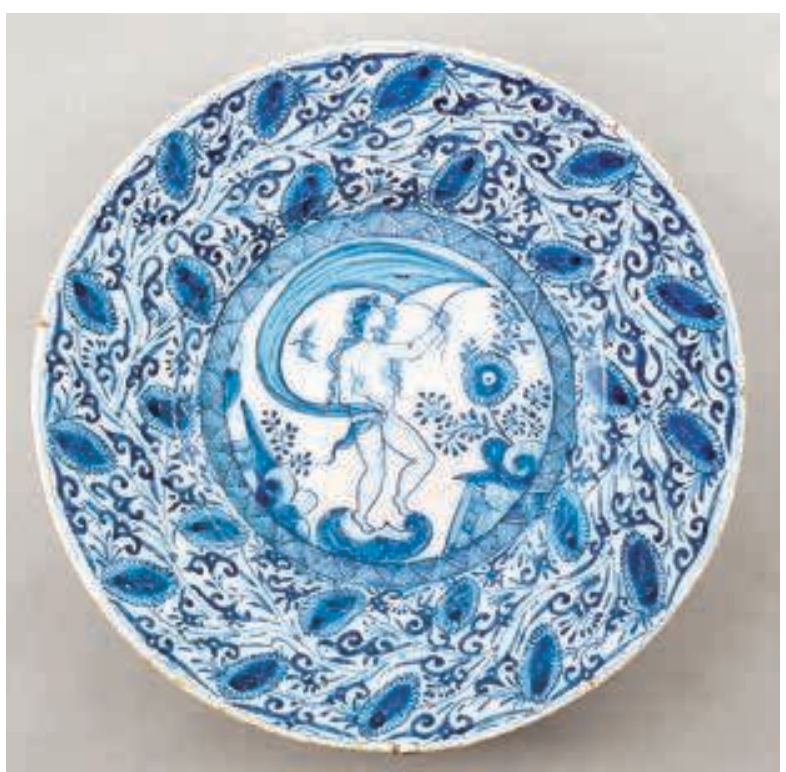

7. Atelier portugais, assiette, $\mathrm{XVII}{ }^{\mathrm{e}}$ siècle, faïence, diam. 36,7 cm, Lisbonne, Museu nacional de arte antiga, inv. n ${ }^{\circ} 2391$ Cer. d'Espagne (Philippe II du Portugal) en 1619 à Lisbonne, et notamment dans sa description de l'arc des potiers ${ }^{18}: l^{\prime}$ Art y est représenté posant sa main gauche sur une roue de potier et tenant dans sa main droite un vase imitant la porcelaine de Chine, le tout accompagné d'un emblème flanqué d'une sentence corroborant l'effective diffusion des productions portugaises : " Les nôtres voyagent aussi vers d'autres régions " (LAVANHA, 1622, fo 30). Si les ateliers lisboètes se sont consacrés à la réalisation de pièces "imitant " les porcelaines, leurs productions ne peuvent cependant pas être réduites à de simples copies. La faune et la flore, sans oublier la palette chromatique appliquée, illustrent des phénomènes de transpositions qui transcendent les modèles et proposent de nouveaux répertoires.

Un autre mécanisme se dessine, fondé sur le mélange des sensibilités par la transmission d'une connaissance ou d'un savoir-faire. Le concept de métissage, étudié avec justesse par Serge Gruzinski ainsi qu'Alessandra Russo (GRUZINSKI, 1999 ; RUSSO, 2013), apparaît ici sousjacent. Toutefois, l'usage délicat et parfois discutable de cette idée suppose la connaissance de deux cultures souches et l'identification de leurs traits respectifs dans l'analyse des objets. La question du mélange des formes est ici analysée à l'aune des migrations artistiques et de leur impact sur les milieux d'accueil. Si on ne conserve que peu de traces des mouvements d'hommes et de femmes natifs des Indes vers l'Europe, le départ d'artistes depuis Lisbonne est mieux documenté. Peintres, sculpteurs, doreurs, faïenciers ont participé aux missions religieuses ou se sont embarqués par-delà les mers en quête de fortune et de succès. La présence d'artistes parmi les missionnaires a été un facteur déterminant dans la diffusion et le mélange des sensibilités. Les congrégations à l'origine de commandes destinées au décor et à l'embellissement des couvents et autres édifices religieux ont joué un rôle non seulement dans le développement de modèles mais aussi dans la formation d'artistes locaux. Au Brésil, deux cas de figure illustrent avec justesse ce phénomène : Agostinho da Piedade (1580-1661) et Agostinho de Jesus (1600-1661). Une fois installé à São Salvador, le premier avait débuté une production de sculptures en terre cuite aux formes 


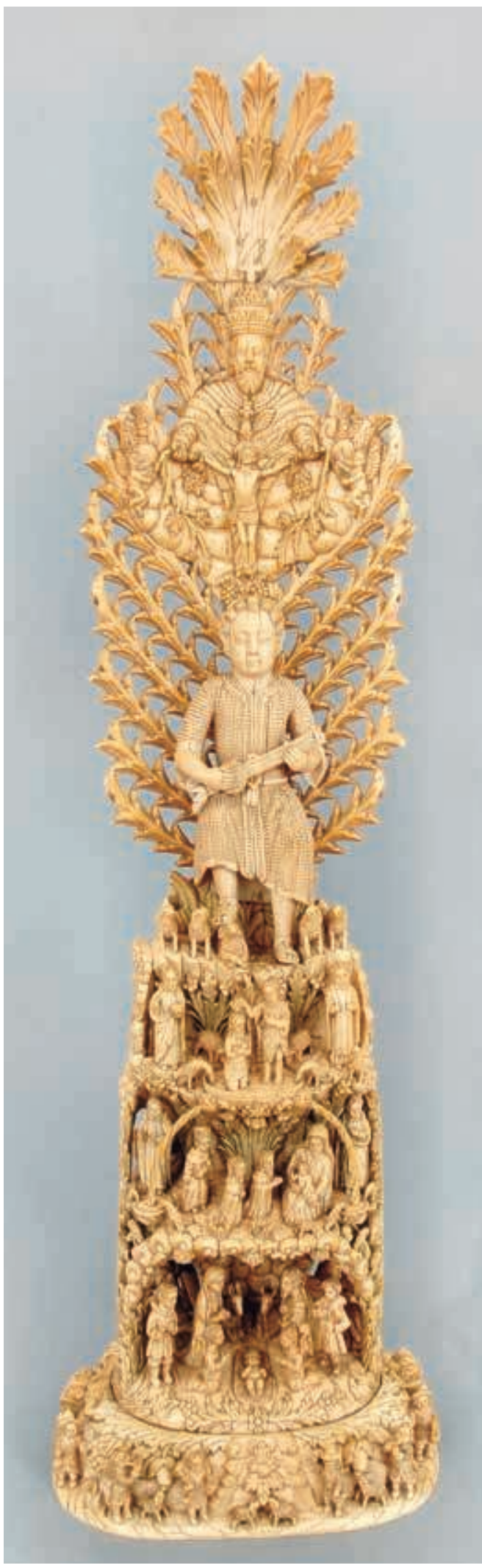

classiques liées à sa formation initiale. Quant au second, il naquit au Brésil et se forma auprès de Piedade. Établi dans la région de São Paulo, il est l'auteur d'une production qui présente une certaine orthodoxie au regard des canons européens appliqués (SILVA NIGRA, 1971 ; SENOS, 2009). La prospérité du Brésil durant la première période coloniale s'est traduite par la construction d'églises où bois doré et sculptures polychromes composaient un décor qui alliait enseignement liturgique et harmonie visuelle. L'importation d'œuvres d'art a perduré dans le temps, avant qu'une production locale puissante et organisée ne se mette en place, à la suite de l'occupation hollandaise entre 1624 et 1654 .

La présence d'artistes peintres lisboètes à Goa est également attestée par le jésuite Manuel Álvares qui réalise en 1562 des peintures pour l'église de Graça de Choran et pour le collège de São Paulo Velho, notamment La Conversion de saint Paul (SERRÃO, 2012a). Plusieurs noms émergent dans la documentation des Archives historiques de Panaji (ou Panjim) : l'ingénieur et architecte Júlio Simão dit aussi Simonis, les sculpteurs canariens Babuxa et maître Santopa, les peintres Aleixo Godinho et João Peres, les orfèvres Jerónimo da Costa, le doreur António da Costa (SERRÃO, 2012a). D'origine portugaise ou Indiens convertis, ces figures récemment découvertes attestent une mobilité humaine dont les fresques et les peintures ornant les chapelles du couvent Santa Mónica sont les témoignages ${ }^{19}$. Les répertoires iconographiques et ornementaux déployés ne sont pas sans évoquer les modèles italo-flamands qui ont été combinés à la culture locale. Le syncrétisme qui se déploie aussi bien en peinture que dans les arts du décor génère des figures et des formes chrétiennes interprétées à la lumière des références hindouistes. Également nées de la volonté d'insinuer un enseignement de la religion par la décoration d'espaces ou le façonnement d'objets, les ivoires de Ceylan matérialisent, eux aussi, le mélange des sensibilités et la réunion des traditions visuelles (fig. 8 ; VASSALO E SILVA, 2013). Ces ivoires ont en commun de présenter une structure compositionnelle similaire aux idoles hindoues. Les sculpteurs tamils à l'origine de ces objets répondaient à la politique de conversion instillée par

8. Atelier de Goa, Enfant Jésus / Menino Bom Pastor XVII ${ }^{\mathrm{e}}$ siècle, ivoire, $72,5 \times 20,5 \mathrm{~cm}$, Vila Viçosa, Fundação Casa de Bragança, inv. n PDVV7229. 
les missionnaires désireux de commander des ouvrages d'une grande lisibilité - respectant les dogmes du concile de Trente - et ne détournant pas les esprits des nouveaux convertis (SERRÃO, 2012b).

L'arrivée des Portugais dans ces contrées a également généré la construction d'une imagerie nouvelle. Au Bénin, la confrontation avec les soldats portugais se matérialise dans la facture singulière de certains ivoires présentant des figures masculines ailées, nues, imberbes, avec le crâne rasé et dotées d'un nez crochu (MAssinG, 2013 ; LOWE, 2017). Ce personnage n'est cependant pas sans rappeler les êtres mythologiques de la tradition $\mathrm{Fon}^{20}$, et notamment le culte de Hon-Dé (fig. 9). On trouve en effet quelques accointances entre ce "faucon-palmier " et le personnage ici représenté : outre ses ailes renvoyant au faucon, un arbre germe, pousse de son nombril, faisant dès lors écho au culte du nombril "hon ", le centre de l'homme, le noyau de la vie (Monsia, 2007) ${ }^{21}$. L'arbre de vie représenté par le palmier " Dé " renvoie à la création originelle symbolisant l'union de l'homme à Dieu et son salut. L'association de ce personnage avec le soldat figurant à ses côtés est pour le moins singulière. Empoignant une

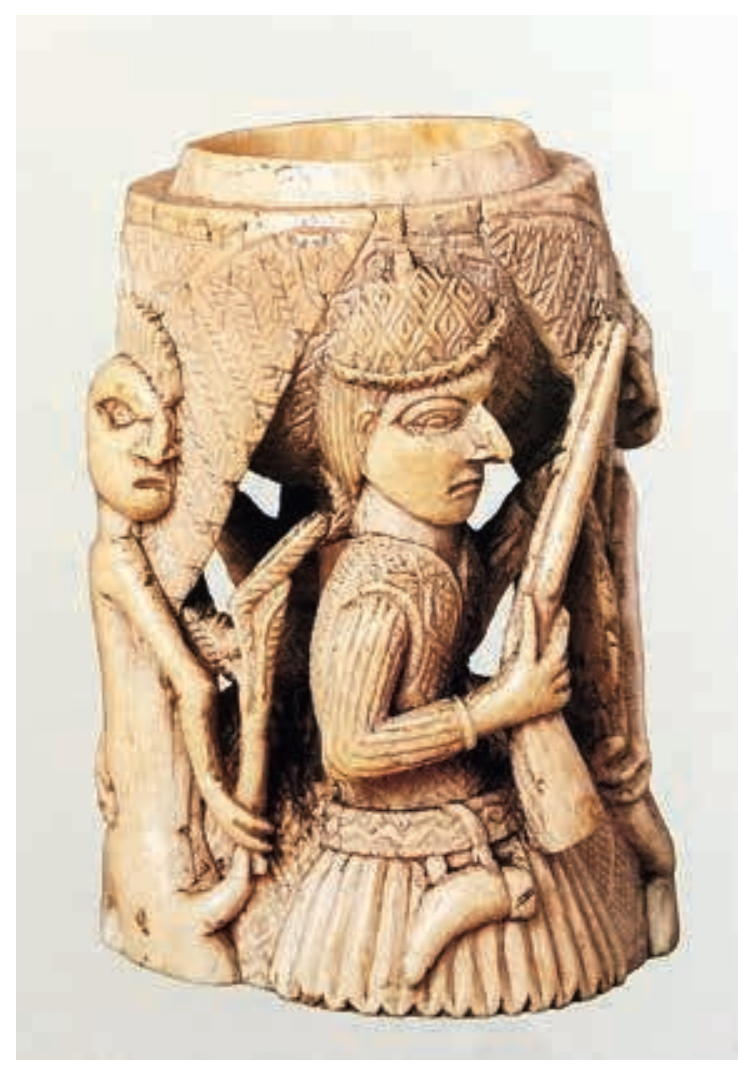

9. Atelier bini-portugais, salière, $x \mathrm{XI} \mathrm{I}^{\mathrm{e}}$ siècle, ivoire, $11,5 \times 8,5 \mathrm{~cm}$, collection particulière. arme à feu, le soldat est vêtu d'une veste, d'une jupe et d'un ceinturon auquel est accroché un poudrier. Son couvre-chef et sa veste sont ornés de « diamants », motifs géométriques issus du répertoire béninois. Le divin et l'humain sont alors réunis. L'image de l'étranger mêlé à la culture locale s'introduit dans les répertoires. Ce phénomène est également visible dans l'art Namban où la présence européenne fut immortalisée sous le pinceau des artistes japonais de l'école Kano (CURVELO, 2009 ; 2015). Les paravents - byōbu en japonais et biombo en portugais - destinés aux élites japonaises mettent en scène l'arrivée des Portugais sur les côtes de l'archipel à bord du kurofune, du « navire noir " (fig. 10) ; en tenue d'apparat, ils sont accompagnés de leurs esclaves africains et de leurs interprètes du Malabar. Mille et un objets intégrés au cortège sont représentés : jarres dites martabans, soieries, épices, animaux (MITSURU, NARUSAWA et al., 2008 ; CURVELO, 2013). Cette mise en scène est également significative d'un changement de point de vue où la vision de l'artiste japonais retranscrit la rencontre de mondes se découvrant l'un l'autre ${ }^{22}$.

Loin d'homogénéiser et d'opposer les productions européennes aux réalisations non-européennes, la notion d'hybridité apporte un nouvel éclairage quant à la valeur tant matérielle que symbolique qu'elles supposent. Dans la lignée des travaux de Peter Burke, l'analyse à la fois fouillée et complexe de ce concept par Carolyn Dean et Dana Leibsohn montre l'adéquation et les limites de cette idée dont l'usage indifférencié peut mener à des contresens ${ }^{23}$. L'union, sur une même surface ou un même support, de traditions visuelles hétérogènes illustrent une reconnaissance de l'autre par l'intégration 


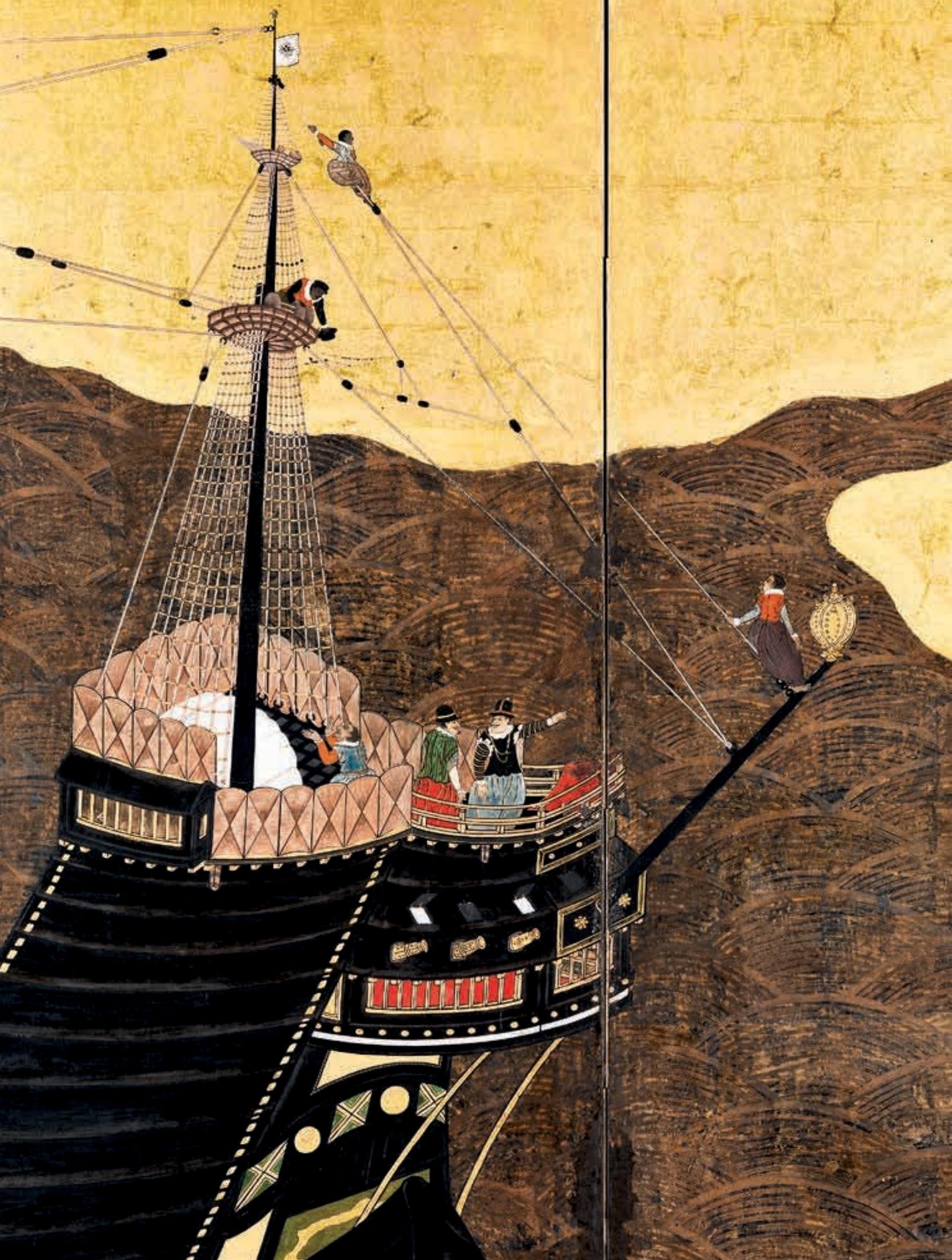


d'éléments issus de sa culture et la remise en question des normes liées au contexte du moment (FERNÁNDEZ-GONZÁLEZ, TRUSTED, 2020a et 2020b). La réception de ces objets et leur usage révèlent les choix opérés par les artistes dans la reconstruction de nouveaux répertoires visuels. Le langage ornemental déployé est, en ce sens, le témoin direct de cette hybridité des formes. La circulation des objets engendre une adaptation des motifs qui démontre toute la capacité des artistes à moduler et à interpréter les répertoires sous de nouvelles modalités entraînant la formation de répertoires ornementaux hybrides. Au-delà du processus d'imitation, la transposition de formes orientales, indiennes ou chinoises dans les langages ornementaux et figuratifs portugais rend compte de processus interprétatifs et adaptatifs inédits (VENTURA TEIXEIRA, 2020a). Daims, biches, chevreuils et cerfs sont ainsi déclinés sur les parements d'azulejos polychromes, dont la facture et le tracé ne sont pas sans rappeler les figures peuplant

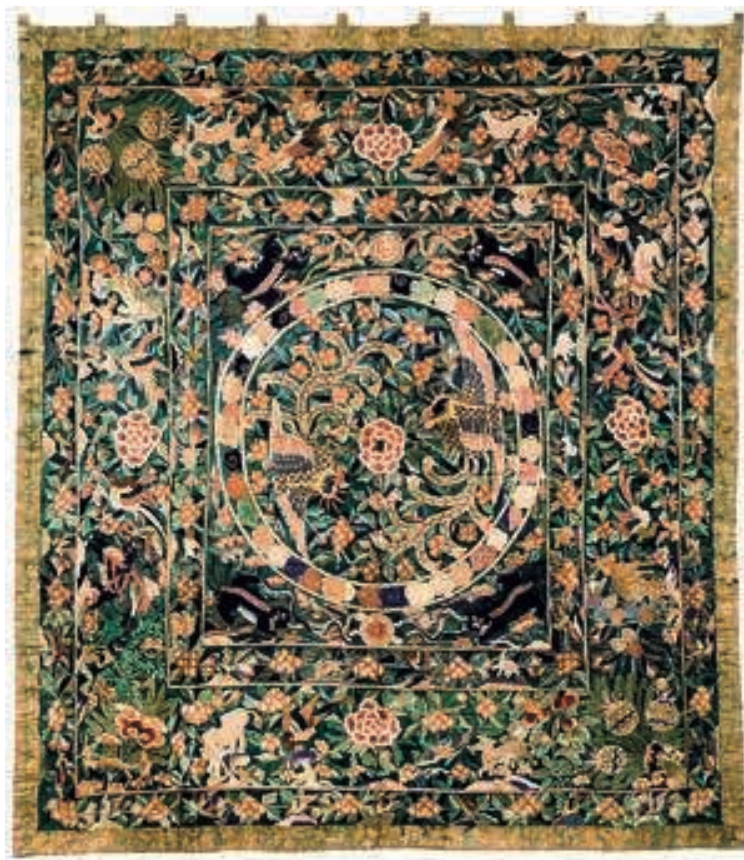

11. Atelier chinois, Pano de armar, $X \mathrm{XII}{ }^{\mathrm{e}}$ siècle, lin, broderie de soie et fil doré, $244 \times 210 \mathrm{~cm}$,

Viseu, Museu nacional Grão Vasco, inv. nº 2899. les fonds d'assiettes de porcelaine, les étoffes indiennes et chinoises ou encore les textiles iraniens (fig. 11; MECO, 1998 ; HALLETT, 2002 ; Monteiro, 2012 ; Nobre Pais, Monteiro, 2013 ; Ventura TeiXeira, 2020b). Si dans la culture chinoise, les cervidés symbolisent la longévité, voire l'immortalité, leur application dans les décors d'autels et autres antependiums céramiques suit un même versant symbolique. Le sens initial n'est pas détourné mais adapté à la culture européenne. Servant de monture au dieu de l'immortalité Shouxing, le cerf est identifié au Christ dans la culture chrétienne. Faisant partie des animaux du paradis de l'arche de Noé ou accompagnant les récits hagiographiques, cet animal n'est pas un motif dénué de sens, simplement apposé ou inséré dans le maillage ornemental des décors d'azulejos. La transposition de ces figures communes aux cultures chrétiennes et taoïstes se fait dans l'harmonie des usages propres à l'univers d'accueil. Les motifs véhiculés par les porcelaines ou les textiles chinois s'inscrivent non seulement dans une histoire du goût mais traduisent aussi des processus de création réfléchis et pensés en fonction des transpositions opérées (FERREIRA, 2013). Les ornements de porcelaine sont loin d'être appliqués au gré des caprices du faïencier. Ils répondent à l'élaboration d'un maillage décoratif religieux qui respecte l'usage de chaque figure ou ornement. Motifs phytomorphes de tradition mudéjare, grotesques hispano-flamands, oiseaux du paradis indo-perse et pagode érigée en arrière-plan (fig. 12) : tels sont les différents motifs composant un parement d'autel émaillé conservé au Museu nacional do azulejo, où le peintre mélange et fait dialoguer plusieurs traditions ornementales dans un souci d'harmonie. D'une grande richesse décorative, les décors d'azulejos matérialisent un phénomène de transculturation où diverses

10. Kano Domi, Biombo (détail), 1593-1601, $172,8 \times 380,8 \mathrm{~cm}$, Lisbonne, Museu nacional de arte antiga, inv. $n^{\circ} 1638$ Mov. esthétiques s'unissent sur une même surface et produisent de nouveaux répertoires (VENTURA 


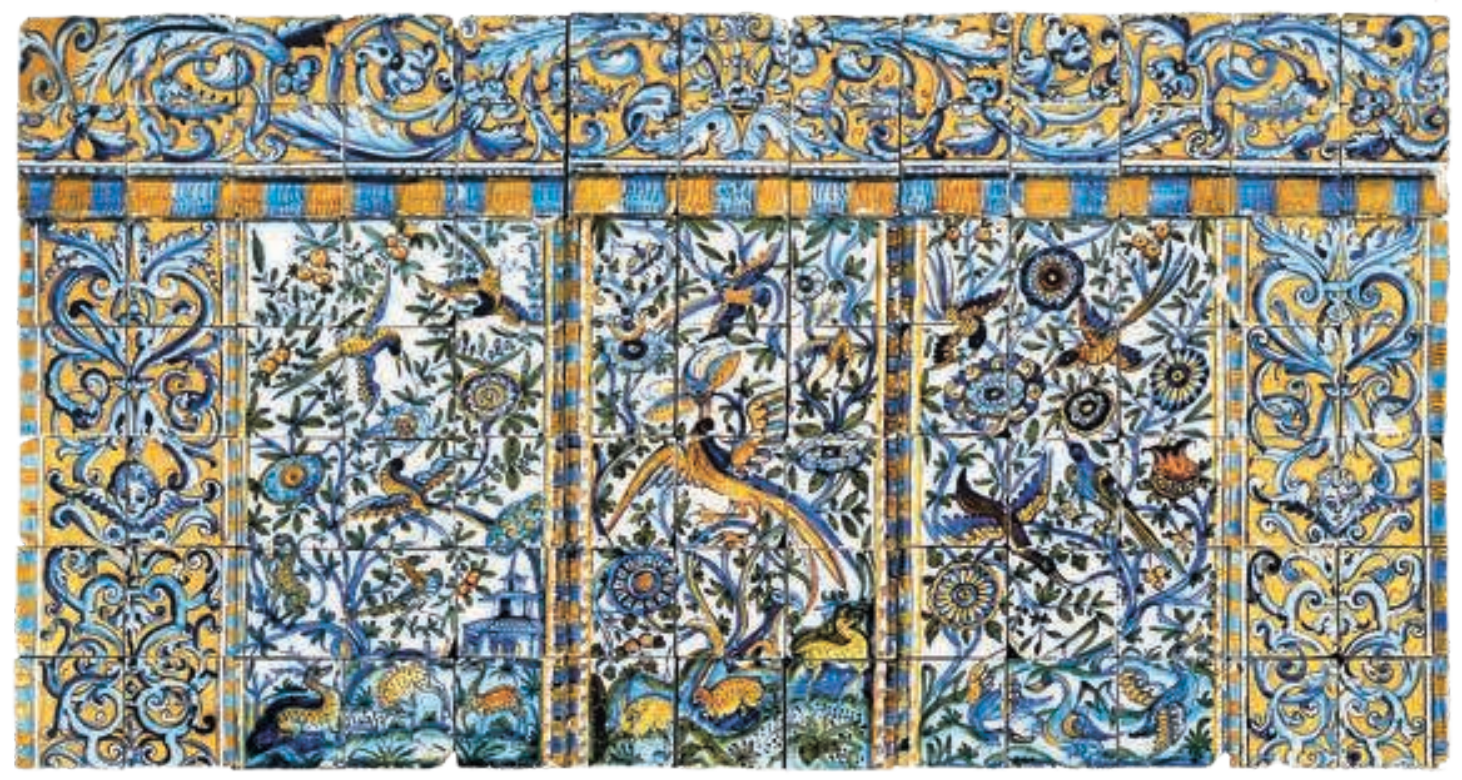

12. Anonyme, atelier de Lisbonne, [Paons], vers 1650 , azulejos polychromes, $92 \times 176 \mathrm{~cm}$, Lisbonne, Museu nacional do azulejo, dépôt museu Machado de Castro (Coimbra), inv. $\mathrm{n}^{\circ} 1438 ; \mathrm{C} 161$.
TEIXEIRA, 2020a). Les ornements appartiennent à différents temps, invoquent différentes mémoires et établissent des liens reposant sur le dialogue des formes. À cette mobilité spatiale des objets et des formes s'ajoute également une mobilité temporelle, où la réminiscence de temps révolus transparaît sous la main des artistes et des artisans.

Les œuvres, les modèles, les motifs sont des entités en constante mutation, témoins de temporalités (BELTING, BUDDENSIEG, 2009). Les arts du décor développés tout au long des XVI et XVII ${ }^{\mathrm{e}}$ siècles non seulement dans les Indes, l'Asie, l'Afrique, l'Orient ou les Amériques mais aussi - et en retour - au Portugal matérialisent les différents visages d'un monde en perpétuel mouvement.

\begin{abstract}
Construite par assises, taillée dans le marbre, coulée dans le bronze, fixée sous le vernis, gravée dans le cuivre ou dans le bois, l'œuvre d'art n'est qu'apparemment immobile. Elle exprime un vœu de fixité, elle est un arrêt, mais comme un moment dans le passé. En réalité, elle naît d'un changement et elle prépare un autre ${ }^{24}$.
\end{abstract}

Les identités culturelles ne sont pas closes et substantielles mais, au contraire, instables, continuellement transformées par les contacts, les conflits et les échanges (LÉVI-STRAUSS, 1977). Les répertoires ornementaux et décoratifs induisent de nouveaux champs interprétatifs où le sens et la signification des formes sont, dans un premier temps, déterminés par les cultures du créateur et du commanditaire et, dans un second temps, par la culture de réception. Ainsi, ce n'est pas tant l'appartenance originelle d'un motif ornemental qui importe mais les processus d'adaptation et les mécanismes de circulation qu'implique la migration d'hommes et d'objets. L'afflux de ces objets répondant à des mobiles commerciaux, religieux ou politiques détermine certes les répertoires mais n'empêche nullement les libertés d'adaptations des modèles. La considération des mécanismes circulatoires s'avère déterminante pour appréhender la genèse d'un objet ou la construction de répertoires appliqués aux arts du décor. 


\section{Céline Ventura Teixeira}

Maîtresse de conférences en histoire de l'art moderne à Aix-Marseille université, Céline Ventura Teixeira est spécialisée dans les arts de la péninsule Ibérique des $\mathrm{XVI}^{\mathrm{e}}$ et $\mathrm{XVII}{ }^{\mathrm{e}}$ siècles. Son ouvrage L'Azulejo, la genèse d'un art : regards croisés entre les ateliers de la péninsule Ibérique (1556-1668), issu de sa thèse de doctorat soutenue à I'université Paris-Sorbonne et récompensée par le Prix de la chancellerie des universités de Paris, a récemment été publié aux éditions Mare et Martin avec le concours du Labex CAP.

\section{NOTES}

1. De 1497 jusqu'au XIXe siècle, cette liaison maritime annuelle est devenue le nerf commercial, financier et stratégique de la couronne portugaise, assurant le transport de biens et d'hommes et consolidant la présence portugaise dans l'espace maritime indien. Voir BOXER, 1961 ; LOPES, FRUTUOSO, GUINOTE, 1992 ; CARREIRA, 1999.

2. GRUZINSKI, (2004) 2006, p. 322.

3. Voir VerísSIMO SERRÃO, 1994 ; BOUZA ÁlVAREZ, 2000 ; SCHAUB, 2001.

4. Dieter Henrich, Konstellationen: Probleme und Debatten am Ursprung der idealistischen Philosophie (1789-1795), Stuttgart, Klett-Cotta, 1991, cité par Sanjay Subrahmanyam, dans sa leçon inaugurale prononcée au Collège de France : Sanjay Subrahmanyam, «Aux origines de l'histoire globale : leçon inaugurale prononcée le jeudi 28 novembre 2013 », Paris, Collège de France, 2014 [DOI : 10.4000/books.cdf.3599].

5. Ces différents systèmes entrent dans le champ épistémologique de I'« histoire globale » ou des « histoires connectées ». Fernand Braudel avait déjà questionné les mécanismes de l'échange et leurs conséquences dans un contexte socio-économique donné, puis Roger Chartier, Sanjay Subrahmanyam ou encore Serge Gruzinski ont offert un certain nombre d'« outils » méthodologiques et théoriques portant un nouvel éclairage sur les acteurs de ces circulations tant matérielles qu'intellectuelles. Voir CHARTIER, 2001 ; SUBRAHMANYAM, (1993) 2013 ; $1994 ; 1997$.

6. L'intérêt pour les expressions artistiques liées aux contacts établis entre l'Asie et l'Europe s'exprime pour la première fois en 1881 avec l'exposition organisée à Londres au South Kensington Museum, «Special Loan Exhibition of Spanish and Portuguese Ornamental Art » qui fut prolongée à Lisbonne au Museu nacional de belas artes e arqueologia (l'actuel Museu nacional de arte antiga) sous le titre "Exposição retrospectiva de arte ornamental portugueza e hespanhola » (SOUSA VITERBO, 1883). À partir des années 1930 et malgré le contexte nationaliste, les quelques études développées par Reynaldo dos Santos, João Couto, Maria Madalena de Cagigal e Silva, Luís Keil, Bernardo Ferrão Tavares e Távora ou Mário Tavares Chicó ont permis de mettre en évidence les relations culturelles et artistiques entre
I'Orient et l'Occident à travers l'identification et l'inventaire des productions artistiques liées à l'aire indienne (LOPES OLIVEIRA, 2011).

\section{Voir DESWARTE-ROSA, 1974 ; 1992.}

8. Les Portugais débarquent accidentellement sur les rives de Ceylan en 1501 et font forte impression au roi qui leur accorde le droit d'établir un fort à Kōtte afin de protéger les marchandises en transit. En maintenant son statut d'unique vassal de dom João III au Sri Lanka, le roi de Kōtte espérait tenir à distance ses rivaux lankais. Cannelle, éléphants et pierres précieuses constituaient le tribut versé, renforçant l'alliance militaire (FLORES, 1998).

9. Ces biens intégrèrent par la suite la collection de son neveu Rodolphe II (1552-1612). Voir GSCHWEND, 1994 ; GSCHWEND, BELTZ, 2010.

10. Voir KARL, 2011 ; 2016 ; Hallett, Pereira, 2007.

11. Les essais de Giorgio Riello et Beverly Lemire sur le commerce du textile indien offrent un nouvel éclairage sur les mécanismes de diffusion à l'échelle internationale (LEMIRE, RIELLO, 2008 ; LEMIRE, 2009 ; RIELLO, 2009).

\section{Voir FerReIRA, OLIVER et al., 2016.}

13. Ce galion assurait l'acheminement des marchandises chinoises contre de l'argent mexicain.

14. À l'image de João de Pedro, achetant ces ouvrages au nom de la reine dona Catarina à Goa, marchands et négociants recevaient les ordres de commandes et renvoyaient l'objet brut ou travaillé au préalable par les ateliers (VASSALO E SILVA, 2000 ; GSCHWEND, 2017).

15. D'après le Tratado da magestade grandeza e abastança da cidade de Lisboa conservé à la Biblioteca nacional de Portugal et écrit par João Brandão en 1552, il existait déjà à Lisbonne soixante fours dédiés à la réalisation d'objets céramique de diverses natures (BRANDAO, [1552] 1990).

16. Les premiers essais de fabrication de porcelaines ont lieu au XVI ${ }^{e}$ siècle à Venise, à Pesaro, à Ferrare ou encore à Florence, foyer à l'origine de la première « porcelaine des Médicis ». Puis, en 1673, les faïenceries de Rouen et de Saint-Cloud tentent l'expérience qui aboutit à la production d'objets de porcelaine tendre (faite d'une pâte à base d'une argile autre que le kaolin). Enfin, en 1708, un gisement de kaolin est découvert en Saxe, annonçant les débuts d'une production européenne de porcelaine dure.

17. Un grand nombre de pièces ont ainsi pris les chemins de Hambourg et d'Amsterdam, où une importante communauté juive d'ascendance portugaise était installée, en particulier dans le quartier de Vlooienburg.

18. Le parcours de l'entrée royale dans les rues de Lisbonne était jalonné d'arcs de triomphe représentants les différents corps de métiers de la ville (arc des orfèvres, arc des peintres, arc des tailleurs, arc des potiers...) mais aussi des communautés (arc des Flamands, arc des Anglais, arc des Allemands...).

19. Le projet de recherche mené par Vítor Serrão et Vanessa Antunes autour de la production artistique de Goa - «De artibus in auream Goa » - a eu le mérite de mettre au jour une documentation inédite issue des archives historiques de Goa. Elle a permis l'identification 
d'artistes d'origine indienne au sein d'un foyer dont la vitalité artistique restait encore méconnue.

20. Population vivant dans le Centre et le Sud du Bénin.

21. Ce culte suppose d'enterrer une partie du cordon ombilical sur lequel est planté un palmier, symbolisant la longévité et la prospérité promises à l'enfant.

22. La représentation de l'« autre » constitue un champ de recherche large récemment étudié par Victor Stoichita (STOICHITA, 2019).

23. DEAN, LeIBSOHN, 2003, p. 6.

24. FOCILLON, (1934) 1943, p. 8.

\section{BIBLIOGRAPHIE SÉLECTIVE}

- AfOnSO, Silva HORTA, 2013 : Luís Urbano Afonso et José da Silva Horta, «Afro-Portuguese Olifants with Hunting Scenes (c. 1490-c. 1540) », Mande Studies, $\mathrm{n}^{\circ} 15,2013$, p. $79-97$.

- Aguiló AlONSO, 1999 : María Paz Aguiló Alonso, "Precisiones sobre el coleccionismo de arte Namban en el siglo XVI ", dans El arte en las cortes de Carlos V y Felipe II, Madrid, CSIC, 1999, p. 151-168.

- AHMED, 1991 : Afzal Ahmed, Indo-Portuguese Trade in Seventeenth Century (1600-1663), New Delhi, Gian Publishing House, 1991.

- AlBUQUeRQUE, (1557) 1973 : Afonso Brás de Albuquerque, Comentários de Afonso de Albuquerque (1557), Joaquim Veríssimo Serrão (éd.), Lisbonne, Imprensa nacional - Casa da Moeda, 1973.

- Alferes PINTO, 2018 : Carla Alferes Pinto, «Entre a Ásia e a Europa: representação e consume artístico da Infanta D. Maria (1521-1577) ", dans Isabel Soares de Albergaria, Duarte Nuno Chaves (dir.), Viagens, produtos e consumos artísticos. O espaço ultramarino português (1450-1900), Lisbonne, CHAM, 2018, p. 9-27.

-

- BAART, 1987 : Jan Baart, « Faiança portuguesa escavada no solo de Amsterdão ", dans idem et Rafael Salinas Calado, Faiança portuguesa (1600-1660), Amsterdam, Amsterdams Historisch Museum, 1987, p. 19-26.

- BAILEY, 2013 : Gauvin Alexander Bailey, «Trasladação e metamorphose dos motivos católicos da China, Japão e Filipinas, 1561-1800 ", dans idem, Jean-Michel Massing et Nuno Vassalo e Silva (dir.), Marfins no império português, Lisbonne, Scribe, 2013, p. 231-290.

- BARreto, Serrão, 2015 : Luís Filipe Barreto, Vítor Serrão, Património cultural chinês em Portugal, Lisbonne, Centro científico e cultural de Macau / Fundação Jorge Álvares, 2015.

- BASSANI, 2000 : Ezio Bassani, African Art and Artefacts in European Collections (1400-1800), Malcolm McLeod (éd.), Londres, British Museum, 2000.

- BELTING, BUDDENSIEG, 2009 : Hans Belting et Andrea Buddensieg (dir.), The Global Art World: Audiences, Markets, and Museums, Ostfildern, Hatje Cantz, 2009. - BÉNAT-TACHOT, GRUZINSKI, 2002 : Louise Bénat-Tachot et Serge Gruzinski (dir.), Passeurs culturels : mécanismes de métissage, actes de colloque (Marne-la-Vallée, université de Marne-la-Vallée, 1998), Paris / Marne-la-Vallée,
Maison des sciences de l'homme / Presses universitaires de Marne-la-Vallée, 2002.

- BERG, 2009 : Maxine Berg, "Quality, Cotton and the Global Luxury Trade », dans Giorgio Riello et Tirthankar Roy (dir.), How India Clothed the World: The World of South Asian Textiles (1500-1850), Leyde / Boston, Brill, 2009, p. 391-414.

- BieDERMANN, 2017 : Zoltán Biedermann, «Diplomatic Ivories: Sri Lankan Caskets and the Portuguese-Asian Exchange in the Sixteenth Century » dans idem, Anne Gerritsen et Giorgio Riello (dir.), Global Gifts: The Material Culture of Diplomacy in Early Modern Eurasia, Cambridge, Cambridge University Press, 2017.

- BOuza Álvarez, 2000 : Fernando Bouza Álvarez, Portugal no tempo dos Filipes: política, cultura, representações (1580-1668), Lisbonne, Cosmos, 2000.

- BOXER, 1961 : Charles Ralph Boxer, "The "Carreira da Índia": Ships, Men, Cargoes, Voyages ", Centro de Estudos Históricos Ultramarinos e as comemorações Henriquinas, Lisbonne, [s.n.], 1961, p. 33-82.

- BRANDÃO, [1552] 1990 : João Brandão, Grandeza e abastança de Lisboa em 1552, José de Felicidade Alves (éd.), Lisbonne, Livros Horizonte, 1990.

- BURKE, 2016 : Peter Burke, Hybrid Renaissance: Culture, Language, Architecture, Budapest, Central European University Press, 2016.

- CARREIRA, 1999 : Ernestine Carreira, "Les naufrages de la Carreira da Índia (XVI - début XIX ${ }^{\mathrm{e}}$ siècle) », Cahiers d'études romanes, no 3, 1999, p. 105-128.

- ChARTIER, 2001 : Roger Chartier, "La conscience de la globalité ", Annales. Histoire, sciences sociales, vol. 56, n 1, 2001, p. 119-123.

- CHAUNU, (1969) 1983 : Pierre Chaunu, L'expansion européenne du XII au XVe siècle (1969), Paris, Presses universitaires de France, 1983.

- CONDE y CeRVANTES, 2014 : José Ignacio Conde y Cervantes, « Las armas reales de España en la porcelana china de exportación », dans Amaya Garritz et Javier Sanchiz Ruiz (dir.) Genealogía, heráldica y documentación, Mexico, Universidad nacional autónoma de México / Instituto de investigaciones históricas, 2014, p. 513-532. - CORREIA, 1918a : Vergílio Correia, "Oleiros e pintores de louça e azulejo, de Lisboa », Atlântida, n² 29-30, mars-avril 1918, p. 531-540.

- CORREIA, 1918b : Vergílio Correia, « Azulejadores e pintores de azulejos, de Lisboa ", A Águia, vol. XIII no 77-78, mai-juin 1918, p. 166-178.

- CRESPO, 2014 : Hugo Miguel Crespo, Jóias da Carreira da Índia, Lisbonne, Fundação Oriente, 2014.

- CRESPO, 2015 : Hugo Miguel Crespo, "Global Interiors on the Rua Nova in Renaissance Lisbon » dans Annemarie Jordan Gschwend et Kate Lowe (dir.), The Global City: On the Streets of Renaissance Lisbon, Londres, Paul Holberton Publishing, 2015, p. 121-139. - CRESPO, 2017 : Hugo Miguel Crespo, « Joalharia entre a Ásia e a Europa », dans GSCHWEND, LOWE, 2017a, p. 216-217.

- CRESPO, 2019 : Hugo Miguel Crespo, «The Plundering of the Ceylonese Royal Treasury, 1551-1553: Its Character, Cost and Dispersal ", dans Michael Bycroft et Sven Dupré (dir.), Gems in the Early Modern World: Materials, Knowledge and Global Trade (1450-1800), Londres, Palgrave Macmillan, 2019, p. 35-64. 
- CRICK, 2017 : Monique Crick, «Sur la route des épices et des porcelaines de la Carreira da India aux Indes néerlandaises, XVe-XVII siècles", dans eadem (dir.) Le Bleu des mers : dialogues entre la Chine, la Perse et I'Europe, cat. exp. (Genève, Fondation Baur - musée des Arts d'Extrême-Orient, 2017-2018), Genève / Milan, Fondation Baur - musée des Arts d'Extrême-Orient / 5 Continents Éditions, 2017, p. 17-21.

- Curvelo, 2008 : Alexandra Curvelo, "The Artistic Circulation between Japan, China and the New-Spain in the 16th and 17th Centuries", Bulletin of Portuguese -Japanese Studies, vol. 16, juin 2008, p. 59-69.

- CuRvelo, 2009 : Alexandra Curvelo, «The Portuguese in Asia during the 16th and 17th Centuries: Economic and Social Dynamics, Artistic and Cultural Experiences", dans Biombos Namban - Namban Screens, Lisbonne, Museu nacional Soares dos Reis / Instituto dos museus e da conservação, 2009, p. 18-42.

- CuRvelo, 2013 : Alexandra Curvelo, "Nanban Art: What's Past in Prologue ", dans Victoria Weston (dir.), Portugal, Jesuits and Japan: Spiritual Beliefs and Earthly Goods, cat. exp. (Boston, McMullen Museum of Art, 2013), Boston, McMullen Museum of Art, 2013, p. 71-78. - Curvelo, 2015 : Alexandra Curvelo, Chefs-d'œuvre des paravents Nanban : Japon-Portugal XVII siècle, Paris, Chandeigne, 2015.

- DaCosta KaufmanN, 2004 : Thomas DaCosta Kaufmann, Toward a Geography of Art, Chicago / Londres, University of Chicago Press, 2004.

- DaCosta KaUfManN, DOSSIN, JOYEUX-PRUNEL, 2015 : Thomas DaCosta Kaufmann, Catherine Dossin et Béatrice Joyeux-Prunel, Circulations in the Global History of Art, Farnham, Ashgate, 2015.

- DEAN, LEIBSOHN, 2003 : Carolyn Dean et Dana Leibsohn, " Hybridity and Its Discontents: Considering Visual Culture in Colonial Spanish America ", Colonial Latin American Review, vol. 12, n 1, 2003, p. 5-35.

- DÉLÉRY, TSAO, 2019 : Claire Déléry et Huei-Chung Tsao (dir.), Un firmament de porcelaines, de la Chine à l'Europe, cat. exp. (Paris, Musée national des arts asiatiques - Guimet, 2019), Paris, Musée national des arts asiatiques - Guimet / Réunion des musées nationaux - Grand Palais, 2019.

- DeSWARTE-ROSA, 1974 : Sylvie Deswarte-Rosa, «Contribution à la connaissance de Francisco de Holanda ", Arquivos do Centro Cultural Português, n 7, 1974, p. 421-429.

- DESWARTE-ROSA, 1992 : Sylvie Deswarte-Rosa, Ideias e imagens em Portugal na época dos descobrimentos: Francisco de Holanda e a teoria da arte, Lisbonne, DIFEL, 1992.

- DIAS, 1995 : Pedro Dias, A viagem das formas: estudos sobre as relações artísticas de Portugal com a Europa, a África, o Oriente e as Américas, Lisbonne, Editorial Estampa, 1995.

- DíAZ, 2010 : Rocío Díaz, Porcelana china para España, Londres, Jorge Welsh Books, 2010.

- Fennetaux, Miller Blaise, OdDO, 2020 : Ariane Fennetaux, Anne-Marie Miller Blaise et Nancy Oddo (dir.), Objets nomades : circulations matérielles, appropriations et formations des identités à l'ère de la première mondialisation (XVI'-XVIII siècles), Turnhout, Brepols, 2020.
- FERNÁNDEZ-GONZÁleZ, TRUSTED, 2020a : Laura Fernández-González et Marjorie Trusted (dir.), "Visual and Spatial Hybridity in the Early Modern Iberian World ", numéro spécial de Renaissance Studies, vol. 34, $n^{\circ} 4$, septembre 2020.

- Fernández-GONZÁlez, TRUSTED, 2020b : Laura Fernández-González et Marjorie Trusted, "Visual and Spatial Hybridity in the Early Modern Iberian World ", Renaissance Studies, vol. 34, no 4, septembre 2020, p. 536-549.

- FERREIRA, 2013 : Maria João Pacheco Ferreira, «Among Birds and Flowers: Repercussions of Chinese Textiles in the Portuguese Azulejo of the 17th Century » dans Alexandra Curvelo (dir.), The Exotic is Never at Home? The Presence of China in the Portuguese Faience and Azulejo (17th-18th Centuries), cat. exp. (Lisbonne, Museu nacional do azulejo, 2014), Lisbonne, Museu nacional do azulejo, 2013, p. 81-95.

- Ferreira, 2014 : Maria João Pacheco Ferreira, «Os "Panos da Índia" em Portugal : integração e consumo dos artigos têxteis asiáticos na sociedade portuguesa dos séculos XVI a XVIII ", dans Actas do IV Congresso de história da arte portuguesa em homenagem a José-Augusto França, Lisbonne, APHA, 2014, p. 72-81.

- FERREIRA, 2016 : Maria João Pacheco Ferreira, « Os limites no gosto: os panos da India nas pragmáticas de 1609 e 1677 ", dans Ana Duarte Rodrigues (dir.), O gosto português na arte, Lisbonne, Scribe, 2016, p. 22-39.

- Ferreira, 2019 : Maria João Pacheco Ferreira, «A propósito das sedas chinesas e a identidade da faiança portuguesa do século XVII ", dans Luxo asiático: porcelana, laca e seda - do consumo à apropriação, cat. exp. (Lisbonne, Museu nacional de arte antiga, 2020), Lisbonne, Museu nacional de arte antiga, 2019, p. 74-85.

- FerReIRA, Oliver et al., 2016 : Maria João Ferreira, Liza Oliver, Maria Ludovica Rosati et Corinne ThépautCabasset, "Les textiles à la période moderne : circulation, échanges et mondialisation", Perspective : actualité en histoire de l'art, $\mathrm{n}^{\circ}$ 1, 2016, p. 21-32 [DOI : 10.4000/perspective.6287].

- FLORES, 1998 : Jorge Manuel Flores, Os Portugueses e o mar de Ceilão: trato, diplomacia e guerra (14981543), Lisbonne, Cosmos, 1998.

- FOCILLON, (1934) 1943 : Henri Focillon, Vie des formes (1934), Paris, Presses universitaires de France, 1943.

\section{$\bullet$}

- GerRITSEN, MCDOWALL, 2012 : Anne Gerritsen et Stephen McDowall, « Material Culture and the Other: European Encounters with Chinese Porcelain, ca. 16501800 ", Global China, numéro spécial de Journal of World History, vol. 23, n 1, mars 2012, p. 87-113.

- GeRRITSEN, RIELLO, 2015 : Anne Gerritsen et Giorgio Riello (dir.), The Global Lives of Things: The Material Culture of Connections in the Early Modern World, Londres, Routledge, 2015.

- GÓIs, 1554 : Damião de Góis, Urbis Olisiponis Descriptio, Évora, André de Burgos, 1554.

- GRUZINSKI, 1999 : Serge Gruzinski, La pensée métisse, Paris, Fayard, 1999.

- GrUZINSKI, (2004) 2006 : Serge Gruzinski, Les Quatre parties du monde : histoire d'une mondialisation (Paris, 2004), Paris, Points, 2006.

- GRUZINSKI, 2015 : Serge Gruzinski, «Art History and Iberian Worldwide Diffusion: Westernization / 
Globalization / Americanization », dans DACOSTA KAUFMANN, DOSSIN, JOYEUX-PRUNEL, 2015, p. 47-58. - GSCHWEND, 1995 : Annemarie Jordan Gschwend, « In the Tradition of Princely Collections: Curiosities and Exotica in the Kunstkammer of Catherine of Austria », Bulletin of the Society for Renaissance Studies, vol. 13, no 1, octobre 1995, p. 48-58.

- GSCHWEND, 1996 : Annemarie Jordan Gschwend, "As maravilhas do Oriente: colecções de curiosidades renascentistas em Portugal », dans Nuno Vassallo e Silva (dir.), A Herança de Rauluchantim, Lisbonne, Museu de São Roque, 1996, p. 82-127.

- GSCHWEND, 2015-2016 : Annemarie Jordan Gschwend, "A Global Thimble in the Renaissance. Regal Needlecases, Sewing Implements and Thimbles from Ceylon for Catherine of Austria, Queen of Portugal ", Museu, IVe série, nº 22, 2015-2016, p. 11-19.

- GSCHWEND, 2017 : Annemarie Jordan Gschwend, "Fazer compras na Rua Nova dos Mercadores », dans GSCHWEND, LOWE, 2017a, p. 134-143.

- GSCHWEND, BeLTZ, 2010 : Annemarie Jordan Gschwend et Johannes Beltz (dir.), Elfenbeine aus Ceylon: Luxusgüter für Katharina von Habsburg (1507-1578), cat. exp. (Zurich, Museum Rietberg, 2011), Zurich, Museum Rietberg, 2010.

- GSCHWEND, LOWE, 2017a: Annemarie Jordan Gschwend, Kate J. P. Lowe (dir.), A cidade global: Lisboa no Renascimento / The Global City: Lisbon in the Renaissance, cat. exp. (Lisbonne, Museu nacional de arte antiga, 2017), Lisbonne, Museu nacional de arte antiga / Imprensa nacional - Casa da Moeda, 2017.

- GSCHWEND, LOWE, 2017b : Annemarie Jordan Gschwend et Kate J. P. Lowe, "Sítios globais da Lisboa renascentista », dans GSCHWEND, LOWE, 2017a, p. 32-59.

- HALLETT, 2002 : Jessica Hallett, « O rouxinol e a rosa: a imagética do amor na arte persa e portuguesa », Oriente, $n^{\circ}$ 4, décembre 2002, p. 3-20.

- Hallett, Pereira, 2007 : Jessica Hallett et Teresa Pacheco Pereira (dir.), O tapete oriental em Portugal: tapete e pintura, séculos XVI-XVIII, cat. exp. (Lisbonne, Museu nacional de arte antiga, 2007), Lisbonne, Museo nacional de arte antiga, 2007.

$\bullet$

- KARL, 2011 : Barbara Karl, " "Marvellous Things are Made with Needles": Bengal Colchas in European Inventories, c. 1580-1630 ", Journal of the History of Collections, vol. 23, $\mathrm{n}^{\circ}$ 2, 2011, p. 301-313.

- KARL, 2016 : Barbara Karl, Embroidered Histories: Indian Textiles for the Portuguese Market during the Sixteenth and Seventeenth Centuries, Vienne, Böhlau Verlag, 2016

- KOPYTOFF, 1986 : Igor Kopytoff, « The Cultural Biography of Things: Commoditisation as Process ", dans Arjun Appadurai (dir.), The Social Life of Things: Commodities in Cultural Perspective, Cambridge, Cambridge University Press, 1986, p. 64-91.

- KRAHE, 2012-2013 : Cinta Krahe, "Chinese Porcelain in Spain during the Habsburg Dynasty », Transactions of the Oriental Ceramic Society, vol. 77, 2012-2013, p. 25-37.

- KRAHL, 2009 : Regina Krahl, «A presença portuguesa nas artes e oficias da China ", dans Encompassing the Globe: Portugal e o mundo nos séculos XVI X XVII, cat. exp.
(Washington, D.C., Arthur M. Sackler Gallery, 2007 ; Lisbonne, Museo nacional de arte antiga, 2009), Lisbonne, Museu nacional de arte antiga, 2009, p. 311-318.

- KUBLER, 1959 : George Kubler, Art and Architecture in Spain and Portugal and their American Dominions (1500-1800), Mitcham, Penguin Books, 1959.

- LAVANHA, 1622 : João Baptista Lavanha, Viagem da catholica real magestade del Rey D. Philippe II N. S ao Reyno de Portugal, Madrid, Thomas lunti, 1622.

- LEMIRE, 2009 : Beverly Lemire, "Revising the Historical Narrative: India, Europe, and the Cotton Trade, c. 1300-1800 ", dans Prasannan Parthasarathi et Giorgio Riello (dir.), The Spinning World: A Global History of Cotton Textiles (1200-1850), Oxford / New York, Oxford University Press, 2009, p. 205-226.

- Lemire, Riello, 2008 : Beverly Lemire et Giorgio Riello, "East \& West: Textiles and Fashion in Early Modern Europe ", Journal of Social History, vol. 41, no 4, 2008, p. 887-916.

- LÉVI-STRAUSS, 1977 : Claude Lévi-Strauss (dir.), L'Identité : séminaire interdisciplinaire, actes du séminaire (Paris, Collège de France, 1974-1975), Paris, Grasset 1977.

- LION-GOLDSCHMIDT, 1984 : Daisy Lion-Goldschmidt, "Les porcelaines chinoises du palais de Santos ", Arts asiatiques, $\mathrm{n}^{\circ} 39,1984$, p. 5-72.

- LOPES, FRUTUOSO, GUINOTE, 1992 : António Lopes, Eduardo Frutuoso et Paulo Guinote, "O movimento da Carreira da Índia nos séculos XVI-XVIII. Revisão e propostas ", Mare Liberum, n 4, décembre 1992, p. 187-265.

- LOPES OliveIRA, 2011 : Rui Lopes Oliveira, "Arte e alteridade: confluências da arte cristã na India, na China e no Japão, séculos XVI a XVIII ", thèse de doctorat, Lisbonne, Faculdade de Belas-Artes da Universidade de Lisboa, 2011, p. 7-25.

- LOWE, 2017 : Kate J. P. Lowe, "Saleiro de Marfim ", dans GSCHWEND, LOWE, 2017a , p. 122-123.

- LUTERBACHER, 2019 : Samuel Luterbacher, "Surfaces for Reflection: Nanban Lacquer in the Iberian World", Angela Vanhaelen et Bronwen Wilson (dir.), "Making Worlds: Art, Materiality, and Early Modern Globalization ", numéro spécial du Journal of Early Modern History, vol. 23, n 2-3, 2019, p. 152-190.

- MANGUCCI, 1996 : António Celso Mangucci, « Olarias de louça e azulejos da freguesia de Santos-o-Velho, dos meados do século XVI aos meados do século XVIII », Al-Madan, $2^{\text {e }}$ série, no 5, octobre 1996, p. 155-161.

- MARK, 2010 : Peter Mark, "On the Misattribution of the Luso-African Ivories: Why Art Historical Scholarship Must Be Based on a Critical Interpretation of Historical Documents ", dans MENDONÇA, REBELO CORREIA, 2010, p. $175-182$.

- MARK, 2009 : Peter Mark, «Portugal na África occidental. Os marfins afro-portugueses », dans Encompassing the Globe : Portugal e o mundo nos séculos XVI e XVII, cat. exp. (Washington, D.C., Arthur M. Sackler Gallery, 2007 ; Lisbonne, Museo nacional de arte antiga, 2009), Lisbonne, Museu nacional de arte antiga, 2009, p. 131-143.

- MARTINS, 2010 : Maria d'Oliveira Martins (dir.), Encomendas Namban: os Portugueses no Japão da idade 
moderna, cat. exp. (Lisbonne, Museu da Fundacão Oriente, 2011), Lisbonne, Fundação Oriente, 2010.

- MASSING, 2009 : Jean Michel Massing, "Escultura em pedra e em marfim na Serra Leoa ", dans Encompassing the Globe: Portugal e o mundo nos séculos XVI e XVII, cat. exp. (Washington, D.C., Arthur M. Sackler Gallery, 2007 ; Lisbonne, Museo nacional de arte antiga, 2009), Lisbonne, Museu nacional de arte antiga, 2009, p. 115-130.

- MASSING, 2013 : Jean Michel Massing, "Os marfins africanos e os portugueses / African Ivories and the Portuguese ", dans idem, Gauvin Alexander Bailey et Nuno Vassallo e Silva (dir.), Marfins no império português, Lisbonne, Scribe, 2013, p. 10-85.

- MECO, 1998 : José Meco, Os frontais de altar quinhentistas e seiscentistas de azulejo: do mudejarismo a influencia oriental, Lisbonne, s.l., 1998.

- Mendes PINTO, 1990 : Maria Helena Mendes Pinto, Lacas Namban em Portugal: presença portuguesa no Japão, Lisbonne, INAPA, 1990.

- MENDONÇA, REBELO CORREIA, 2010 : Isabel Mayer Godinho Mendonça et Ana Paulo Rebelo Correia (dir.), As artes decorativas e a expansão portuguesa: imaginário e viagem, actes du colloque international (Lisbonne, museu de Artes Decorativas da Fundação Ricardo do Espírito Santo Silva, 2008), Lisbonne, Fundação Ricardo do Espírito Santo Silva, 2010.

- Mitsuru, Narusawa et al., 2008 : Sakamoto Mitsuru, Katsushi Narusawa et al., Nanban byōbu shūsei. Catalogue Raisonné of the Namban Screens, Tokyo, Chūō Kōron Bijutsu Shuppan, 2008.

- MONSIA, 2007 : Marc Monsia, Introduction au panthéon vodoun, Jean-Dominique Burton (photographies), Cotonou, Fondation Zinsou, 2007.

- MONTEIRO, 2012 : João Pedro Monteiro (dir.), Um gosto português: o uso do azulejo no século XVII, cat. exp. (Lisbonne, Museu nacional do azulejo, 2012), Lisbonne, Athena, 2012.

\section{$\bullet$}

- NOBRE PAIS, 2012 : Alexandre Nobre Pais, " "Fabricado no Reino Lusitano o que antes nos vendeu tão caro a China": a produção de faiança em Lisboa, entre os reinados de Filipe II e D. João V », thèse de doctorat, Lisbonne, Universidade Católica Portuguesa, 2012.

- NOBRE PAIS, MONTEIRO, 2013 : Alexandre Nobre Pais et João Pedro Monteiro, "O exótico na faiança e no azulejo portugueses do século XVII ", dans Alexandra Curvelo (dir.), The Exotic is Never at Home? The Presence of China in the Portuguese Faience and Azulejo (17th-18th Centuries), cat. exp. (Lisbonne, Museu nacional do azulejo, 2013-2014), Lisbonne, Museu nacional do azulejo, 2013, p. 59-79.

\section{$\bullet$}

- Oliveira e COSTA, 1993 : João Paulo Oliveira e Costa, Portugal e o Japão: o século Namban, Lisbonne, Imprensa nacional - Casa da Moeda, 1993.

- Oliveira E COSTA, 2007 : João Paulo Oliveira e Costa, Dom Manuel I, 1469-1521: um príncipe do Renascimento, Lisbonne, Círculo dos Leitores, 2007.

\section{-}

- PINTO DE MATOS, 1994 : Maria Antónia Pinto de Matos, "A porcelana chinesa: referencia essencial na faiança portuguesa de seiscentos », dans eadem et João Pedro
Monteiro (dir.), A influência oriental na cerâmica portuguesa do século XVII, cat. exp. (Lisbonne, Museu nacional do azulejo, 1994), Lisbonne / Milan, Lisboa Capital Europeia da Cultura '94 / Electa, 1994.

\section{$\bullet$}

- RIELLO, 2009 : Giorgio Riello, « The Globalization of Cotton Textiles: Indian Cottons, Europe, and the Atlantic World, 1600-1850 », dans Prasannan Parthasarathi et Giorgio Riello (dir.), The Spinning World: A Global History of Cotton Textiles (1200-1850), Oxford / New York, Oxford University Press, 2009, p. 261-287.

- RUSSO, 2013 : Alessandra Russo, L'Image intraduisible : une histoire métisse des arts en Nouvelle-Espagne (15001600), Dijon, Les presses du réel, 2019.

- RUSSO, 2020 : Alessandra Russo, « Lights on the Antipodes: Francisco de Holanda and an Art History of the Universal », The Art Bulletin, vol. 102, n 4, 2020, p. 37-65.

- SANTOS SIMÕES, 1971 : João Miguel dos Santos Simões, Azulejaria em Portugal no século XVII, Lisbonne, fondation Calouste Gulbenkian, 1971, 2 vol.

- SCHAUB, 2001 : Jean-Frédéric Schaub, Portugal na monarquia hispânica (1580-1640), Lisbonne, Livros Horizonte, 2001.

-SENOS, 2009 : Nuno Senos, "As artes no Brasil antes da idade de Ouro ", dans Encompassing the Globe: Portugal e o mundo nos séculos XVI e XVII, cat. exp. (Washington, D.C., Arthur M. Sackler Gallery, 2007 ; Lisbonne, Museo nacional de arte antiga, 2009), Lisbonne, Museu nacional de arte antiga, 2009, p. 177-184.

- SERRÃO, 2012a : Vítor Serrão, "Pintura e devoção em Goa no tempo dos Filipes: o mosteiro de Santa Mónica no "Monte Santo" (c. 1606-1639) e os seus artistas ", Oriente, $\mathrm{n}^{\circ}$ 20, 2012, p. 11-50.

- SERRÃO, 2012b : Vítor Serrão, « Impactos do concílio de Trento na arte portuguesa entre o maneirismo e o barroco ", dans José Pedro Paiva (dir.), O Concilío de Trento em Portugal e nas suas conquistas. Olhares novos, Lisbonne, Centro de Estudos de história religiosa da Universidade Católica Portuguesa, 2020, p. 103-132.

- Silva Nigra, 1971 : Clemente M. da Silva Nigra, Os dois escultores: Frei Agostinho da Piedade, Frei Agostinho de Jesus e o Arquitecto Frei Macario de São João, Salvador, Universidad Federal de Bahia, 1971.

- SOUSA VITERBO, 1883 : Francisco Marques de Sousa Viterbo, A exposição de arte ornamental: notas ao catálogo, Lisbonne, Imprensa nacional, 1883.

- STOICHITA, 2019 : Victor Stoichita, Darker Shades: The Racial Other in Early Modern Art, Londres, Reaktion Books, 2019.

-SUBRAHMANYAM, (1993) 2013 : Sanjay Subrahmanyam, L'Empire portugais d'Asie, Marie-José Capelle (trad. fr.), Paris, Points, 2013 [éd. orig. : The Portuguese Empire in Asia 1500-1700: A Political and Economic History, Londres, Longman, 1993].

- SubrahmANYAM, 1994 : Sanjay Subrahmanyam, "Writing History "Backwards": Southeast Asian History (and the Annales) at the Crossroads ", Studies in History, vol. 10, n 1, 1994, p. 131-145.

- SUBRAHMANYAM, 1997 : Sanjay Subrahmanyam, "Connected Histories: Notes towards a Reconfiguration of Early Modern Eurasia », Modern Asian Studies, vol. 31, no 3, 1997, p. 735-762. 
- THORNTON, 1992 : John Thornton, Africa and Africans in the Making of the Atlantic World (1400-1800), Cambridge, Cambridge University Press, 1992.

- THORNTON, 2009 : John Thornton, "Relações lusoafricanas (1500-1750) », Encompassing the Globe: Portugal e o mundo nos séculos XVI e XVII, cat. exp. (Washington, D.C., Arthur M. Sackler Gallery, 2007 ; Lisbonne, Museo nacional de arte antiga, 2009), Lisbonne, Museu nacional de arte antiga, 2009, p. 104-114.

- TRNEK, VASSALO E SILVA, 2001 : Helmut Trnek et Nuno Vassalo e Silva (dir.) Exotica: The Portuguese Discoveries and the Renaissance Kunstkammer, cat. exp. (Vienne, Kunsthistorisches Museum, 2002), Lisbonne, fondation Calouste Gulbenkian, 2001.

- VANHAELEN, WILSON, 2019 : Angela Vanhaelen et Bronwen Wilson, "Introduction - Making Worlds: Art, Materiality, and Early Modern Globalization », eaedem, "Making Worlds: Art, Materiality, and Early Modern Globalization », numéro spécial du Journal of Early Modern History, vol. 23, n 2-3, 2019, p. 108-113.

- VASSALO E SILVA, 2000 : Nuno Vassalo e Silva, «Missions and Merchants: Christian Art in Macao ", Oriental Art, vol. 46, no 3, 2000), p. 84-91.

- VASSALO E SILVA, 2013 : Nuno Vassalo e Silva, " "Engenho e Primor": a arte do marfim no Ceilão / "Ingenuity and Excellence": Ivory Art in Ceylon », dans idem, Gauvin Alexander Bailey et Jean Michel Massing (dir.), Marfins no império português, Lisbonne, Scribe, 2013, p. 87-141. - VENTURA TEIXEIRA, 2019 : Céline Ventura Teixeira, L'Azulejo, la genèse d'un art : regards croisés sur les ateliers de la péninsule Ibérique au temps des Philippe (1556-1668), Paris, Éditions Mare et Martin, 2019.

- Ventura TeiXeIRA, 2020a : Céline Ventura Teixeira, "A Palimpsest of Ornaments: The Art of Azulejo as a Hybrid Language ", dans FERNÁNDEZ-GONZÁLEZ, TRUSTED, 2020a, p. 593-623.

- VENTURA TEIXEIRA, 2020b: «Des tapis de soie aux tapis de faïence : I'incorporation d'une flore nouvelle venue $\mathrm{d}^{\prime}$ Orient et des Indes dans les panneaux d'azulejos du XVII siècle", dans FenNeTAUX, MILler Blaise, OdDO, 2020, p. 188-201.

- VERÍSSIMO SERRÃO, 1994 : Joaquim Veríssimo Serrão, O tempo dos Filipes em Portugal e no Brasil (1580-1668), Lisbonne, Edições Colibri, 1994. 